\title{
Thermocapillary motion of a fluid droplet parallel to two plane walls
}

\author{
Huan J. Keh * , Po Y. Chen, Li S. Chen \\ Department of Chemical Engineering, National Taiwan University, Taipei 106-17, Taiwan, ROC
}

Received 15 May 2001; received in revised form 13 January 2002

\begin{abstract}
The steady thermocapillary migration of a fluid droplet located between two infinite parallel plane walls is studied theoretically in the absence of fluid inertia and thermal convection. The imposed temperature gradient is constant and parallel to the two plates, and the droplet is assumed to retain a spherical shape. The plane walls may be either insulated or prescribed with the far-field temperature distribution. The presence of the neighboring walls causes two basic effects on the droplet velocity: first, the local temperature gradient on the droplet surface is enhanced or reduced by the walls, thereby speeding up or slowing down the droplet; secondly, the walls increase viscous retardation of the moving droplet. To solve the thermal and hydrodynamic governing equations, the general solutions are constructed from the fundamental solutions in both the rectangular and spherical coordinate systems. The boundary conditions are enforced first at the plane walls by the Fourier transforms and then on the droplet surface by a collocation technique. Numerical results for the thermocapillary migration velocity of the droplet relative to that under identical conditions in an unbounded medium are presented for various values of the relative viscosity and thermal conductivity of the droplet as well as the relative separation distances between the droplet and the two plates. For the special cases of thermocapillary motions of a spherical droplet parallel to a single plate and on the central plane of a slit, the collocation results agree well with the approximate analytical solutions obtained by using a method of reflections. The presence of the lateral walls can reduce or enhance the droplet velocity, depending upon the relative transport properties of the droplet, the relative droplet-wall separation distances, and the thermal boundary condition at the walls. In general, the boundary effect on thermocapillary migration is quite complicated and relatively weak in comparison with that on sedimentation. (c) 2002 Elsevier Science Ltd. All rights reserved.
\end{abstract}

Keywords: Thermocapillary motion; Fluid sphere; Parallel walls; Boundary effects

* Corresponding author. Fax: +886-2-2362-3040.

E-mail address: huan@ccms.ntu.edu.tw (H.J. Keh). 


\section{Introduction}

A droplet of one fluid, when suspended in a second, immiscible fluid possessing a temperature gradient, will move in the direction of the gradient. The physical explanation of this movement is that the temperature gradient produces a gradient of interfacial tension or Marangoni force along the droplet surface, which drags fluid and propels the droplet toward regions where its interfacial tension would be reduced (usually, hot regions). In addition to its importance from a fundamental point of view, this thermocapillary effect has become a subject of current interest with the development of the orbiting spacecraft and opportunities for experimenting and manufacturing under near-weightless conditions. For example, the removal of unwanted or wanted gas bubbles or liquid droplets from a liquid phase by thermocapillary forces will be encountered during the processing of materials (such as high technology glass and alloy mixtures) in the reduced gravity environment provided by an orbiting laboratory.

The thermocapillary migration of gas bubbles was first demonstrated experimentally by Young et al. (1959). They also theoretically calculated the migration velocity of a spherical fluid droplet of radius $a$ placed in an infinite expanse of suspending fluid of viscosity $\eta$, with a prescribed linear temperature distribution $T_{\infty}(\mathbf{x})$. If the droplet is sufficiently small that effects of inertia and convection of energy are negligible, its velocity $\mathbf{U}_{0}$ is related to the constant temperature gradient $\nabla T_{\infty}$ by the formula

$$
\mathbf{U}_{0}=\frac{2}{\left(2+k^{*}\right)\left(2+3 \eta^{*}\right)}\left(-\frac{\partial \gamma}{\partial T}\right) \frac{a}{\eta} \nabla T_{\infty},
$$

where $\partial \gamma / \partial T$ is the variation of the interfacial tension $\gamma$ between the droplet and the continuous phase with respect to the local temperature $T$ (with a typical value of $10^{-4} \mathrm{~N} \mathrm{~m}^{-1} \mathrm{~K}^{-1}$ ), and $k^{*}$ and $\eta^{*}$ are the ratios of thermal conductivities and viscosities, respectively, between the internal and external fluids. In (1), all the physical properties are assumed to be constant except for the interfacial tension, which is assumed to vary linearly with temperature. The thermocapillary migration velocity of a single gas bubble can be evaluated by (1) taking the limiting values $k^{*}=0$ and $\eta^{*}=0$.

Eq. (1) serves only for a droplet in continuous phases that extend to infinity in all directions. However, in practical applications of thermocapillary motion, droplets usually are not isolated and will move in the presence of neighboring droplets and/or boundaries (Meyyappan et al., 1981, 1983; Anderson, 1985; Acrivos et al., 1990; Morton et al., 1990; Satrape, 1992; Loewenberg and Davis, 1993a,b; Kasumi et al., 2000). During the past two decades, much progress has been made in the theoretical analysis concerning the applicability of (1) for a fluid particle in a variety of bounded systems. Through an exact representation in spherical bipolar coordinates, Meyyappan et al. (1981) and Sadhal (1983) solved the quasisteady problem of thermocapillary motion of a spherical gas bubble normal to an infinite planar solid or free fluid surface of constant temperature. Later, Meyyappan and Subramanian (1987) examined the thermocapillary motion of a gas bubble parallel to a rigid plane surface on which the far-field temperature gradient was imposed. In both cases, the bubble velocity was found to decrease monotonically relative to its isolated value given by (1) for motions close to the boundary.

Extending the analysis by Meyyappan et al. (1981) for a gas bubble, Barton and Subramanian (1990) and Chen and Keh (1990) determined the thermocapillary migration velocity of a fluid 
droplet in the direction perpendicular to a nearby isothermal planar solid or free surface. Analytical solutions of this problem in asymptotic forms were obtained by using a method of reflections (Chen and Keh, 1990; Chen, 1999) and a lubrication approach (Loewenberg and Davis, 1993b). The effect of a planar solid surface on fluid drops undergoing thermocapillary motion normal to it has also been investigated experimentally (Barton and Subramanian, 1991) and was found to be in good agreement with the predictions from the quasisteady analyses. Chen et al. (1991) used a boundary-collocation method to solve for the axisymmetric thermocapillary motion of a spherical drop within a long circular insulated tube. They found that the fluid sphere in the tube always moves slower than it does in an infinite medium as a result of the lateral wall-drop thermal and hydrodynamic interactions, and the thermocapillary velocity is a monotonically decreasing function of the ratio of the sphere to the tube diameter for fixed values of $k^{*}$ and $\eta^{*}$. At constant values of $\eta^{*}$ and the sphere-to-tube diameter, the migration velocity of the drop in the tube relative to the isolated value increases as $k^{*}$ decreases, because a greater portion of energy is conducted through the relatively conductive gap between the drop and the insulated tube wall which creates a larger interfacial tension gradient. In addition to the above-mentioned studies for the boundary effects on the thermocapillary motion of fluid spheres, the migration of a deformable drop normal to an isothermal rigid plane due to thermocapillarity has also been examined (Ascoli and Leal, 1990).

The objective of this article is to obtain exact numerical solutions and approximate analytical solutions for the thermocapillary motions of a spherical fluid droplet parallel to a single plane wall and to two plane walls at an arbitrary position between them. The plane walls may be either insulated or prescribed with the linear far-field temperature distribution. The effects of inertia as well as thermal convection are neglected. For the case of a droplet with a relatively low thermal conductivity undergoing thermocapillary migration near insulated plane walls or of a droplet with a relatively high conductivity undergoing thermocapillary movement near plane walls prescribed with the far-field temperature distribution, the heat conduction around the droplet will generate larger temperature gradients on the droplet surface relative to those in an infinite medium. These gradients enhance the thermocapillary migration velocity, although their action will be retarded by the viscous interaction of the migrating droplet with the walls. Both effects of this thermal enhancement and the hydrodynamic retardation increase as the ratios of the radius of the droplet to its distances from the walls increase. Determining which effect is overriding at small dropletwall gap widths is a main target of this study.

\section{Analysis}

We consider the steady thermocapillary migration of a spherical fluid droplet of radius $a$ in an immiscible fluid parallel to two infinite plane walls whose distances from the center of the droplet are $b$ and $c$, as shown in Fig. 1 . Here $(x, y, z),(\rho, \phi, z)$, and $(r, \theta, \phi)$ denote the rectangular, circular cylindrical, and spherical coordinate systems, respectively, and the origin of coordinates is chosen at the droplet center. A linear temperature field $T_{\infty}(\mathbf{x})$ with a uniform thermal gradient $E_{\infty} \mathbf{e}_{x}$ (equal to $\nabla T_{\infty}$ ) is imposed in the surrounding fluid far away from the droplet, where $\mathbf{e}_{x}$ is the unit vector in the $x$ direction. The capillary number $\eta U_{0} / \gamma$ (where $U_{0}=\left|\mathbf{U}_{0}\right|$ is given by (1)) is assumed to be sufficiently small so that interfacial tension maintains the spherical shape of the droplet 


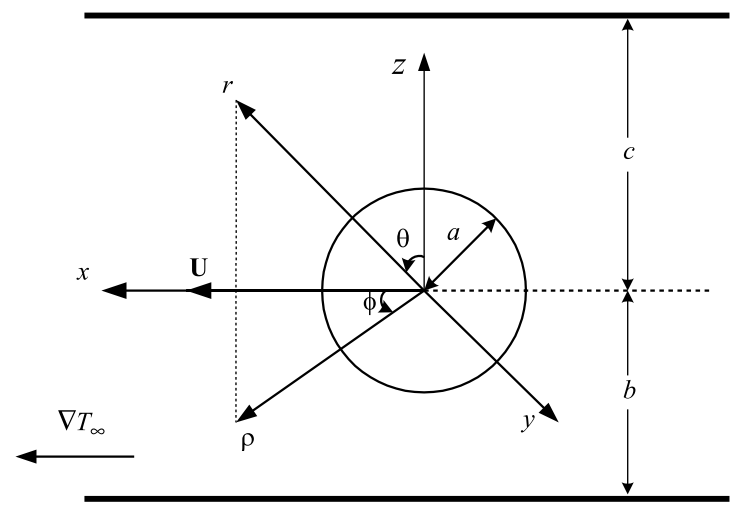

Fig. 1. Geometrical sketch for the thermocapillary motion of a spherical droplet parallel to two plane walls at an arbitrary position between them.

during the confined thermocapillary migration. Gravitational and natural-convection effects are ignored. The objective is to determine the correction to (1) for the droplet velocity due to the presence of the plane walls.

Before determining the thermocapillary migration velocity of the droplet, the temperature and velocity fields in both internal and external fluid phases need to be found.

\subsection{Temperature distribution}

The Marangoni number (Peclet number) of the system is assumed to be small. Hence, the equation of energy governing the temperature distribution $T(\mathbf{x})$ for the external fluid of constant thermal conductivity $k$ is the Laplace equation,

$$
\nabla^{2} T=0 \quad(r \geqslant a)
$$

For the temperature field $T_{1}(\mathbf{x})$ inside the droplet, one has

$$
\nabla^{2} T_{1}=0 \quad(r \leqslant a) .
$$

The boundary conditions require that the temperature and the normal component of heat flux be continuous at the droplet surface and that the temperature field far away from the droplet approach the undisturbed values. Thus,

$$
\begin{aligned}
& r=a: \quad T=T_{1}, \quad k \frac{\partial T}{\partial r}=k_{1} \frac{\partial T_{1}}{\partial r}, \\
& z=c,-b: \quad \frac{\partial T}{\partial z}=0, \\
& \rho \rightarrow \infty: \quad T=T_{0}+E_{\infty} x,
\end{aligned}
$$

where $k_{1}$ is the thermal conductivity of the droplet and $T_{0}$ is the (undisturbed) temperature at the droplet center. Note that the boundary conditions given by (3c) apply for the case of two insulated plane walls. For the case of thermocapillary motion of a droplet parallel to two plane walls 
prescribed with a linear temperature profile consistent with the far-field distribution, (3c) should be replaced by

$$
z=c,-b: \quad T=T_{0}+E_{\infty} x .
$$

For the special case of $k_{1}=k$, both (3c) and (3e) lead to the same linear temperature field for the whole fluid phases as for the far field.

Since the governing equations and boundary conditions are linear, one can express the external temperature distribution $T$, which is symmetric with respect to $y$ and antisymmetric with respect to $x$, as the superposition

$$
T=T_{\mathrm{w}}+T_{\mathrm{s}}
$$

Here, $T_{\mathrm{w}}$ is a double Fourier integral solution of (2a) in rectangular coordinates that represents the disturbance produced by the plane walls plus the undisturbed temperature field and is given by

$$
T_{\mathrm{w}}=T_{0}+E_{\infty} x+E_{\infty} \int_{0}^{\infty} \int_{0}^{\infty}\left(X \mathrm{e}^{\kappa z}+Y \mathrm{e}^{-\kappa z}\right) \sin (\alpha x) \cos (\beta y) \mathrm{d} \alpha \mathrm{d} \beta,
$$

where $X$ and $Y$ are unknown functions of separation variables $\alpha$ and $\beta$, and $\kappa=\left(\alpha^{2}+\beta^{2}\right)^{1 / 2}$. The second term on the right-hand side of (4), $T_{\mathrm{s}}$, is a solution of (2a) in spherical coordinates representing the disturbance generated by the droplet and is given by an infinite series in harmonics,

$$
T_{\mathrm{s}}=E_{\infty} \sum_{n=1}^{\infty} R_{n} r^{-n-1} P_{n}^{1}(\mu) \cos \phi
$$

where $P_{n}^{1}$ is the associated Legendre function of order $n$ and degree one, $\mu$ is used to denote $\cos \theta$ for brevity, and $R_{n}$ are unknown constants. Note that a solution of $T$ of the form given by (4)-(6) immediately satisfies the boundary condition at infinity in (3d). Since the temperature is finite for any position in the interior of the droplet, the solution to (2b) can be written as

$$
T_{1}=T_{0}+E_{\infty} \sum_{n=1}^{\infty} \bar{R}_{n} r^{n} P_{n}^{1}(\mu) \cos \phi,
$$

where $\bar{R}_{n}$ are unknown constants.

A brief conceptual description of the solution procedure to determine $X, Y, R_{n}$, and $\bar{R}_{n}$ is given below to help follow the mathematical development. At first, boundary conditions (given by ( $3 \mathrm{c}$ ) or (3e)) are exactly satisfied on the plane walls by using the Fourier transforms. This permits the unknown functions $X$ and $Y$ to be determined in terms of the coefficients $R_{n}$. Then, the boundary conditions in $(3 \mathrm{a}, \mathrm{b})$ on the surface of the droplet can be satisfied at discrete points by making use of the collocation method (O'Brien, 1968; Ganatos et al., 1980; Chen et al., 1991; Keh and Chen, 1993), and the solution of the collocation matrix provides numerical values for the coefficients $R_{n}$ and $\bar{R}_{n}$.

Substituting the temperature distribution $T$ given by (4)-(6) into the boundary conditions in (3c) or (3e) and applying the Fourier sine and cosine inversions on the variables $x$ and $y$, respectively, lead to a solution for the functions $X$ and $Y$ in terms of the coefficients $R_{n}$. After the substitution of this solution into (5) and utilization of the integral representations of the modified Bessel functions of the second kind, the temperature distribution $T$ can be expressed as 


$$
T=T_{0}+E_{\infty} x+E_{\infty} \sum_{n=1}^{\infty} R_{n} \delta_{n}^{(1)}(r, \mu) \cos \phi,
$$

where the functions $\delta_{n}^{(1)}(r, \mu)$ are defined by (B.1a) in Appendix B. Applying the boundary conditions given by $(3 \mathrm{a}, \mathrm{b})$ to $(7)$ and $(8)$ yields

$$
\begin{aligned}
& \sum_{n=1}^{\infty}\left[R_{n} \delta_{n}^{(1)}(a, \mu)-\bar{R}_{n} a^{n} P_{n}^{1}(\mu)\right]=-a\left(1-\mu^{2}\right)^{1 / 2}, \\
& \sum_{n=1}^{\infty}\left[R_{n} \delta_{n}^{(2)}(a, \mu)-\bar{R}_{n} k^{*} n a^{n-1} P_{n}^{1}(\mu)\right]=-\left(1-\mu^{2}\right)^{1 / 2},
\end{aligned}
$$

where the definition of functions $\delta_{n}^{(2)}(r, \mu)$ is given by (B.1b) and $k^{*}=k_{1} / k$. Note that the definite integrals in $\delta_{n}^{(1)}$ and $\delta_{n}^{(2)}$ must be performed numerically.

To satisfy the conditions in (9) exactly along the entire surface of the droplet would require the solution of the entire infinite array of unknown constants $R_{n}$ and $\bar{R}_{n}$. However, the collocation technique enforces the boundary conditions at a finite number of discrete points on the halfcircular generating arc of the droplet (from $\theta=0$ to $\pi$ ) and truncates the infinite series in (7) and (8) into finite ones. If the spherical boundary is approximated by satisfying the conditions of (3a,b) at $M$ discrete points on its generating arc, the infinite series in (7) and (8) are truncated after $M$ terms, resulting in a system of $2 M$ simultaneous linear algebraic equations in the truncated form of (9). This matrix equation can be numerically solved to yield the $2 M$ unknown constants $R_{n}$ and $\bar{R}_{n}$ required in the truncated form of (7) and (8) for the temperature distribution. The accuracy of the boundary-collocation/truncation technique can be improved to any degree by taking a sufficiently large value of $M$. Naturally, as $M \rightarrow \infty$ the truncation error vanishes and the overall accuracy of the solution depends only upon the numerical integration required in evaluating the matrix elements:

\subsection{Fluid velocity distribution}

With knowledge of the solution for the temperature distribution on the droplet surface which drives the migration, we can now proceed to find the flow field. The fluids inside and outside the droplet are assumed to be incompressible and Newtonian. Owing to the low Reynolds number, the fluid motion caused by the thermocapillary migration of the droplet is governed by the Stokes equations,

$$
\begin{aligned}
& \eta \nabla^{2} \mathbf{v}-\nabla p=\mathbf{0}, \quad \nabla \cdot \mathbf{v}=0 \quad(r \geqslant a), \\
& \eta_{1} \nabla^{2} \mathbf{v}_{1}-\nabla p_{1}=\mathbf{0}, \quad \nabla \cdot \mathbf{v}_{1}=0 \quad(r \leqslant a) .
\end{aligned}
$$

Here, $\mathbf{v}_{1}(\mathbf{x})$ and $\mathbf{v}(\mathbf{x})$ are the fluid velocity fields for the flow inside the droplet and for the external flow, respectively, $p_{1}(\mathbf{x})$ and $p(\mathbf{x})$ are the corresponding dynamic pressure distributions, and $\eta_{1}$ is the viscosity of the droplet.

The boundary conditions for the fluid velocity at the droplet surface (Young et al., 1959; Anderson, 1985), on the plane walls, and far removed from the droplet are

$$
r=a: \quad \mathbf{e}_{r} \cdot(\mathbf{v}-\mathbf{U})=0, \quad \mathbf{v}=\mathbf{v}_{1},
$$




$$
\begin{aligned}
& r=a: \quad\left(\mathbf{I}-\mathbf{e}_{r} \mathbf{e}_{r}\right) \mathbf{e}_{r}:\left(\boldsymbol{\tau}-\tau_{1}\right)=-\frac{\partial \gamma}{\partial T}\left(\mathbf{I}-\mathbf{e}_{r} \mathbf{e}_{r}\right) \cdot \nabla T, \\
& z=c,-b: \mathbf{v}=\mathbf{0}, \\
& \rho \rightarrow \infty: \quad \mathbf{v}=\mathbf{0} .
\end{aligned}
$$

Here, $\gamma(\theta, \phi)$ is the local interfacial tension for the droplet; $\tau=\eta\left[\nabla \mathbf{v}+(\nabla \mathbf{v})^{T}\right]$ and $\tau_{1}=\eta_{1}$ $\left[\nabla \mathbf{v}_{1}+\left(\nabla \mathbf{v}_{1}\right)^{T}\right]$ are viscous stress tensors for the external flow and the flow inside the droplet, respectively; $\mathbf{e}_{r}$ together with $\mathbf{e}_{\theta}$ and $\mathbf{e}_{\phi}$ are the unit vectors in spherical coordinates; $\mathbf{I}$ is the unit dyadic; $\mathbf{U}=U \mathbf{e}_{x}$ is the thermocapillary migration velocity of the droplet to be determined. Note that $\partial \gamma / \partial T$ is assumed to be constant on the scale of the droplet radius and $\nabla T$ can be evaluated from the temperature distribution given by (8) with coefficients determined from (9). For the asymmetric problem as $b \neq c$, the assumption that $\mathbf{U}$ and $\nabla T_{\infty}$ are in the same direction is justified in the absence of inertia.

In view of the linearity of the governing equations and boundary conditions, the external velocity field $\mathbf{v}$ can be decomposed into two contributions (Ganatos et al., 1980),

$$
\mathbf{v}=\mathbf{v}_{\mathrm{w}}+\mathbf{v}_{\mathrm{s}}
$$

Here, $\mathbf{v}_{\mathrm{w}}$ is a solution of (10) in rectangular coordinates that represents the disturbance produced by the plane walls and is given by

$$
\mathbf{v}_{\mathrm{w}}=v_{\mathrm{w} x} \mathbf{e}_{x}+v_{\mathrm{w} y} \mathbf{e}_{y}+v_{\mathrm{w} z} \mathbf{e}_{z}
$$

where $\mathbf{e}_{x}, \mathbf{e}_{y}$, and $\mathbf{e}_{z}$ are the unit vectors in rectangular coordinates, and $v_{\mathrm{w} x}, v_{\mathrm{w} y}$, and $v_{\mathrm{w} z}$ are the double Fourier integrals,

$$
\begin{aligned}
& v_{\mathrm{w} x}=\int_{0}^{\infty} \int_{0}^{\infty} D_{1}(\alpha, \beta, z) \cos (\alpha x) \cos (\beta y) \mathrm{d} \alpha \mathrm{d} \beta, \\
& v_{\mathrm{w} y}=\int_{0}^{\infty} \int_{0}^{\infty} D_{2}(\alpha, \beta, z) \sin (\alpha x) \sin (\beta y) \mathrm{d} \alpha \mathrm{d} \beta \\
& v_{\mathrm{w} z}=\int_{0}^{\infty} \int_{0}^{\infty} D_{3}(\alpha, \beta, z) \sin (\alpha x) \cos (\beta y) \mathrm{d} \alpha \mathrm{d} \beta .
\end{aligned}
$$

In (15)

$$
\begin{aligned}
& D_{1}=\left[X^{*}\left(1+\frac{\alpha^{2}}{\kappa} z\right)-X^{* *} \frac{\alpha \beta}{\kappa} z-X^{* * *} \alpha z\right] \mathrm{e}^{\kappa z}+\left[Y^{*}\left(1-\frac{\alpha^{2}}{\kappa} z\right)+Y^{* *} \frac{\alpha \beta}{\kappa} z-Y^{* * *} \alpha z\right] \mathrm{e}^{-\kappa z} \\
& D_{2}=\left[-X^{*} \frac{\alpha \beta}{\kappa} z+X^{* *}\left(1+\frac{\beta^{2}}{\kappa} z\right)+X^{* * *} \beta z\right] \mathrm{e}^{\kappa z}+\left[Y^{*} \frac{\alpha \beta}{\kappa} z+Y^{* *}\left(1-\frac{\beta^{2}}{\kappa} z\right)+Y^{* * *} \beta z\right] \mathrm{e}^{-\kappa z} \\
& D_{3}=\left[X^{*} \alpha z-X^{* *} \beta z+X^{* * *}(1-\kappa z)\right] \mathrm{e}^{\kappa z}+\left[Y^{*} \alpha z-Y^{* *} \beta z+Y^{* * *}(1+\kappa z)\right] \mathrm{e}^{-\kappa z}
\end{aligned}
$$

where the starred $X$ and $Y$ are unknown functions of separation variables $\alpha$ and $\beta$, and $\kappa=$ $\left(\alpha^{2}+\beta^{2}\right)^{1 / 2}$. 
The second part of $\mathbf{v}$, denoted by $\mathbf{v}_{\mathrm{s}}$, is a solution of (10) in spherical coordinates representing the disturbance generated by the droplet and is given by

$$
\mathbf{v}_{\mathrm{s}}=v_{\mathrm{sx}} \mathbf{e}_{x}+v_{\mathrm{sy}} \mathbf{e}_{y}+v_{\mathrm{sz}} \mathbf{e}_{z}
$$

where

$$
\begin{aligned}
& v_{\mathrm{s} x}=\sum_{n=1}^{\infty}\left(A_{n} A_{n}^{\prime}+B_{n} B_{n}^{\prime}+C_{n} C_{n}^{\prime}\right), \\
& v_{\mathrm{s} y}=\sum_{n=1}^{\infty}\left(A_{n} A_{n}^{\prime \prime}+B_{n} B_{n}^{\prime \prime}+C_{n} C_{n}^{\prime \prime}\right), \\
& v_{\mathrm{s} z}=\sum_{n=1}^{\infty}\left(A_{n} A_{n}^{\prime \prime \prime}+B_{n} B_{n}^{\prime \prime \prime}+C_{n} C_{n}^{\prime \prime \prime}\right) .
\end{aligned}
$$

In (18), the primed $A_{n}, B_{n}$, and $C_{n}$ are functions of position involving associated Legendre functions of $\mu$ or $\cos \theta$ defined by (2.6) of Ganatos et al. (1980) and $A_{n}, B_{n}$, and $C_{n}$ are unknown constants. Note that the boundary condition at infinity in (12e) is immediately satisfied by a solution of the form given by (13)-(18).

The solution to (11) for the internal velocity field can be expressed as

$$
\mathbf{v}_{1}=v_{1 r} \mathbf{e}_{r}+v_{1 \theta} \mathbf{e}_{\theta}+v_{1 \phi} \mathbf{e}_{\phi},
$$

where

$$
\begin{aligned}
& v_{1 r}=\sum_{n=1}^{\infty} n P_{n}^{1}(\mu)\left(\bar{C}_{n} r^{n-1}+\bar{A}_{n} r^{n+1}\right) \cos \phi \\
& v_{1 \theta}=\sum_{n=1}^{\infty}\left[\bar{B}_{n} r^{n} P_{n}^{1}(\mu)\left(1-\mu^{2}\right)^{-1 / 2}-\left(1-\mu^{2}\right)^{1 / 2} \frac{\mathrm{d} P_{n}^{1}(\mu)}{\mathrm{d} \mu}\left(\bar{C}_{n} r^{n-1}-\bar{A}_{n} \frac{n+3}{n+1} r^{n+1}\right)\right] \cos \phi, \\
& v_{1 \phi}=\sum_{n=1}^{\infty}\left[\bar{B}_{n} r^{n}\left(1-\mu^{2}\right)^{1 / 2} \frac{\mathrm{d} P_{n}^{1}(\mu)}{\mathrm{d} \mu}-\left(1-\mu^{2}\right)^{-1 / 2} P_{n}^{1}(\mu)\left(\bar{C}_{n} r^{n-1}-\bar{A}_{n} \frac{n+3}{n+1} r^{n+1}\right)\right] \sin \phi,
\end{aligned}
$$

and $\bar{A}_{n}, \bar{B}_{n}$, and $\bar{C}_{n}$ are unknown constants. A solution of this form satisfies the requirement that the velocity is finite for any position within the droplet.

As was the case with the solution for the temperature distribution, the determination of the starred $X$ and $Y$ functions as well as the coefficients $A_{n}, B_{n}, C_{n}, \bar{A}_{n}, \bar{B}_{n}$, and $\bar{C}_{n}$ is undertaken by a two-step procedure. First, the boundary conditions of (12d) is exactly satisfied on the plane walls by using the Fourier transforms. Then, the conditions in (12a,b) and (12c) are satisfied numerically at collocation points on the droplet surface.

Application of the boundary conditions in (12d) to (13)-(18) and utilization of the Fourier sine and cosine inversions on the variables $x$ and $y$ lead to a solution for $D_{1}, D_{2}$, and $D_{3}$ in terms of the coefficients $A_{n}, B_{n}$, and $C_{n}$. Substituting this solution back into (15) and using the integral representations of the modified Bessel functions of the second kind, one obtains a new expression from (13)-(18) for the external fluid velocity in terms of $A_{n}, B_{n}$, and $C_{n}$,

$$
\mathbf{v}=v_{x} \mathbf{e}_{x}+v_{y} \mathbf{e}_{y}+v_{z} \mathbf{e}_{z},
$$


where

$$
\begin{aligned}
& v_{x}=\sum_{n=1}^{\infty}\left[A_{n}\left(A_{n}^{\prime}+\alpha_{n}^{\prime}\right)+B_{n}\left(B_{n}^{\prime}+\beta_{n}^{\prime}\right)+C_{n}\left(C_{n}^{\prime}+\gamma_{n}^{\prime}\right)\right] \\
& v_{y}=\sum_{n=1}^{\infty}\left[A_{n}\left(A_{n}^{\prime \prime}+\alpha_{n}^{\prime \prime}\right)+B_{n}\left(B_{n}^{\prime \prime}+\beta_{n}^{\prime \prime}\right)+C_{n}\left(C_{n}^{\prime \prime}+\gamma_{n}^{\prime \prime}\right)\right] \\
& v_{z}=\sum_{n=1}^{\infty}\left[A_{n}\left(A_{n}^{\prime \prime \prime}+\alpha_{n}^{\prime \prime \prime}\right)+B_{n}\left(B_{n}^{\prime \prime \prime}+\beta_{n}^{\prime \prime \prime}\right)+C_{n}\left(C_{n}^{\prime \prime \prime}+\gamma_{n}^{\prime \prime \prime}\right)\right] .
\end{aligned}
$$

Here, the primed $\alpha_{n}, \beta_{n}$, and $\gamma_{n}$ are functions of position in the form of integration (which must be performed numerically) defined by (C1) of Ganatos et al. (1980).

The boundary conditions that remain to be satisfied are those on the droplet surface. Substituting (8) and (19)-(22) into (12a,b) and (12c), one obtains

$$
\begin{aligned}
& \sum_{n=1}^{\infty}\left[A_{n} A_{n}^{*}(a, \mu, \phi)+B_{n} B_{n}^{*}(a, \mu, \phi)+C_{n} C_{n}^{*}(a, \mu, \phi)\right]=U\left(1-\mu^{2}\right)^{1 / 2} \cos \phi, \\
& \sum_{n=1}^{\infty}\left[A_{n} A_{n}^{*}(a, \mu, \phi)+B_{n} B_{n}^{*}(a, \mu, \phi)+C_{n} C_{n}^{*}(a, \mu, \phi)\right] \\
& -\sum_{n=1}^{\infty}\left[\bar{C}_{n} n a^{n-1} P_{n}^{1}(\mu)+\bar{A}_{n} n a^{n+1} P_{n}^{1}(\mu)\right] \cos \phi=0, \\
& \sum_{n=1}^{\infty}\left[A_{n} A_{n}^{* *}(a, \mu, \phi)+B_{n} B_{n}^{* *}(a, \mu, \phi)+C_{n} C_{n}^{* *}(a, \mu, \phi)\right]-\sum_{n=1}^{\infty}\left[\bar{B}_{n} a^{n}\left(1-\mu^{2}\right)^{-1 / 2} P_{n}^{1}(\mu)\right. \\
& \left.-\bar{C}_{n} a^{n-1}\left(1-\mu^{2}\right)^{1 / 2} \frac{\mathrm{d} P_{n}^{1}}{\mathrm{~d} \mu}-\bar{A}_{n} \frac{n+3}{n+1} a^{n+1}\left(1-\mu^{2}\right)^{1 / 2} \frac{\mathrm{d} P_{n}^{1}}{\mathrm{~d} \mu}\right] \cos \phi=0, \\
& \sum_{n=1}^{\infty}\left[A_{n} A_{n}^{* * *}(a, \mu, \phi)+B_{n} B_{n}^{* * *}(a, \mu, \phi)+C_{n} C_{n}^{* * *}(a, \mu, \phi)\right]-\sum_{n=1}^{\infty}\left[\bar{B}_{n} a^{n}\left(1-\mu^{2}\right)^{1 / 2} \frac{\mathrm{d} P_{n}^{1}}{\mathrm{~d} \mu}\right. \\
& \left.-\bar{C}_{n} a^{n-1}\left(1-\mu^{2}\right)^{-1 / 2} P_{n}^{1}(\mu)-\bar{A}_{n} \frac{n+3}{n+1} a^{n+1}\left(1-\mu^{2}\right)^{-1 / 2} P_{n}^{1}(\mu)\right] \sin \phi=0, \\
& \sum_{n=1}^{\infty}\left\{\left(\frac{\partial}{\partial r}-\frac{1}{r}\right)\left[A_{n} A_{n}^{* *}(r, \mu, \phi)+B_{n} B_{n}^{* *}(r, \mu, \phi)+C_{n} C_{n}^{* *}(r, \mu, \phi)\right]\right. \\
& \left.-\frac{\left(1-\mu^{2}\right)^{1 / 2}}{r} \frac{\partial}{\partial \mu}\left[A_{n} A_{n}^{*}(r, \mu, \phi)+B_{n} B_{n}^{*}(r, \mu, \phi)+C_{n} C_{n}^{*}(r, \mu, \phi)\right]\right\}_{r=a} \\
& -\eta^{*} \sum_{n=1}^{\infty}\left[\bar{B}_{n}(n-1) a^{n-1} P_{n}^{1}(\mu)\left(1-\mu^{2}\right)^{-1 / 2}-\bar{C}_{n} 2(n-1) a^{n-2}\left(1-\mu^{2}\right)^{1 / 2} \frac{\mathrm{d} P_{n}^{1}(\mu)}{\mathrm{d} \mu}\right. \\
& \left.-\bar{A}_{n} \frac{n(n+2)}{n+1} a^{n}\left(1-\mu^{2}\right)^{1 / 2} \frac{\mathrm{d} P_{n}^{1}(\mu)}{\mathrm{d} \mu}\right] \cos \phi=-\frac{E_{\infty}}{\alpha \eta} \frac{\partial \gamma}{\partial T}\left[a \mu+\sum_{n=1}^{\infty} R_{n} \delta_{n}^{(3)}(a, \mu)\right] \cos \phi,
\end{aligned}
$$




$$
\begin{aligned}
\sum_{n=1}^{\infty}\{ & \left(\frac{\partial}{\partial r}-\frac{1}{r}\right)\left[A_{n} A_{n}^{* * *}(r, \mu, \phi)+B_{n} B_{n}^{* * *}(r, \mu, \phi)+C_{n} C_{n}^{* * *}(r, \mu, \phi)\right] \\
& \left.+\frac{\left(1-\mu^{2}\right)^{-1 / 2}}{r} \frac{\partial}{\partial \phi}\left[A_{n} A_{n}^{*}(r, \mu, \phi)+B_{n} B_{n}^{*}(r, \mu, \phi)+C_{n} C_{n}^{*}(r, \mu, \phi)\right]\right\}_{r=a} \\
- & \eta^{*} \sum_{n=1}^{\infty}\left[\bar{B}_{n}(n-1) a^{n-1}\left(1-\mu^{2}\right)^{1 / 2} \frac{\mathrm{d} P_{n}^{1}(\mu)}{\mathrm{d} \mu}-\bar{C}_{n} 2(n-1) a^{n-2}\left(1-\mu^{2}\right)^{-1 / 2} P_{n}^{1}(\mu)\right. \\
& \left.-\bar{A}_{n} \frac{n(n+2)}{n+1} a^{n}\left(1-\mu^{2}\right)^{-1 / 2} P_{n}^{1}(\mu)\right] \sin \phi=\frac{E_{\infty}}{\alpha \eta} \frac{\partial \gamma}{\partial T}\left[-a\left(1-\mu^{2}\right)^{1 / 2}-\sum_{n=1}^{\infty} R_{n} \delta_{n}^{(1)}(a, \mu)\right] \sin \phi
\end{aligned}
$$

Here, the function $\delta_{n}^{(3)}(r, \mu)$ in (23e) is defined by (B.1c), the definitions of the starred $A_{n}, B_{n}$, and $C_{n}$ functions are given by (B.4a)-(B.4i) and $\eta^{*}=\eta_{1} / \eta$. The first $M$ coefficients $R_{n}$ have been determined through the procedure given in the previous subsection.

Careful examination of (23a)-(23f) shows that the solution of the coefficient matrix generated is independent of the $\phi$ coordinate of the boundary points on the surface of the sphere $r=a$. Thus, these relations can be satisfied by utilizing the collocation technique presented for the solution of the temperature field. At the droplet surface, (23a)-(23f) are applied at $N$ discrete points (values of $\theta$ between 0 and $\pi$ ) and the infinite series in (20) and (22) are truncated after $N$ terms. This generates a set of $6 N$ linear algebraic equations for the $6 N$ unknown coefficients $A_{n}, B_{n}, C_{n}, \bar{A}_{n}, \bar{B}_{n}$, and $\bar{C}_{n}$. The fluid velocity field is completely obtained once these coefficients are solved for a sufficiently large value of $N$. Note that the definite integrals in (23a)-(23f) after the substitution of (B.4) must be performed numerically.

\subsection{Derivation of the droplet velocity}

The drag force exerted by the external fluid on the spherical droplet can be determined from (Ganatos et al., 1980)

$$
\mathbf{F}=-8 \pi \eta A_{1} \mathbf{e}_{x}
$$

This expression shows that only the lowest-order coefficient $A_{1}$ contributes to the hydrodynamic force acting on the droplet.

Because the droplet is freely suspended in the surrounding fluid, the net force exerted on the droplet must vanish. Applying this constraint to (24), one has,

$$
A_{1}=0 \text {. }
$$

To determine the thermocapillary migration velocity $U$ of the droplet, (25) and the $6 N$ algebraic equations resulting from (23a)-(23f) are to be solved simultaneously.

\section{Results and discussion}

The solution for the thermocapillary motion of a spherical droplet parallel to two plane walls at an arbitrary position between them, obtained by using the boundary-collocation technique de- 
scribed in the previous section, is presented in this section. The system of linear algebraic equations to be solved for the coefficients $R_{n}$ and $\bar{R}_{n}$ is constructed from (9), while that for $A_{n}, B_{n}, C_{n}$, $\bar{A}_{n}, \bar{B}_{n}$, and $\bar{C}_{n}$ is composed of (23a)-(23f). All the numerical integrations to evaluate the starred $\delta_{n}$, $A_{n}, B_{n}$, and $C_{n}$ functions were done by the 80-point Gauss-Laguerre guadrature. The numerical calculations were performed by using a DEC 3000/600 workstation.

When specifying the points along the semicircular generating arc of the sphere (with a constant value of $\phi$ ) where the boundary conditions are to be exactly satisfied, the first points that should be chosen are $\theta=0$ and $\pi$, since these points define the projected area of the droplet normal to the direction of motion and control the gaps between the droplet and the neighboring plates. In addition, the point $\theta=\pi / 2$ is also important. However, an examination of the systems of linear algebraic equations in (9) and (23a)-(23f) shows that the matrix equations become singular if these points are used. To overcome this difficulty, these points are replaced by closely adjacent points, i.e., $\theta=\delta, \pi / 2-\delta, \pi / 2+\delta$, and $\pi-\delta$ (Ganatos et al., 1980). Additional points along the boundary are selected as mirror-image pairs about the plane $\theta=\pi / 2$ to divide the two quarter arcs of the droplet into equal segments. The optimum value of $\delta$ in this work is found to be $0.1^{\circ}$, with which the numerical results of the droplet velocity converge satisfactorily. In selecting the boundary points, any value of $\phi$ may be used except for $\phi=0, \pi / 2$, and $\pi$ since the matrix equation (23a)-(23f) is singular for these values.

\subsection{Motion parallel to a single plane wall}

The collocation solutions for the terminal velocity of a fluid droplet undergoing thermocapillary migration parallel to a plane wall (with $c \rightarrow \infty$ ) for different values of the parameters $k^{*}, \eta^{*}$, and $a / b$ are presented in Table 1 for the cases of an insulated wall and a wall with the imposed farfield temperature gradient. The velocity for the thermocapillary motion of an identical droplet in an infinite fluid, $U_{0}$, given by (1), is used to normalize the boundary-corrected values. All of the results obtained under this collocation scheme converge satisfactorily to at least the significant figures shown in the table. The accuracy and convergence behavior of the truncation technique is principally a function of the ratio $a / b$. For the most difficult case with $a / b=0.999$, the numbers of collocation points $M=36$ and $N=36$ are sufficiently large to achieve this convergence.

In Appendix A, an approximate solution for the same thermocapillary motion as that considered here is also obtained by using a method of reflections. The droplet velocity near a lateral plate is given by (A.11), which is a power series expansion in $\lambda(=a / b)$. The values of the wallcorrected normalized thermocapillary mobility calculated from this asymptotic solution, with the $\mathrm{O}\left(\lambda^{8}\right)$ term neglected, are also listed in Table 1 for comparison. It can be seen that the asymptotic formula of (A.11) from the method of reflections agrees very well with the exact results as long as $\lambda \leqslant 0.8$; the errors in all cases are less than $1.3 \%$. However, accuracy of (A.11) begins to deteriorate, as expected, when the relative spacing between the droplet and the plane wall becomes small (say, $\lambda \geqslant 0.9$ ). In general, the formula of (A.11) overestimates the thermocapillary migration velocity of the droplet when $\lambda$ is close to unity.

The exact numerical solutions for the normalized velocity $U / U_{0}$ of a spherical droplet undergoing thermocapillary motion parallel to a plane wall as a function of $a / b$ are depicted in Figs. 2 and 3 for various values of $k^{*}$ and $\eta^{*}$. It can be seen that the wall-corrected normalized thermocapillary mobility of the droplet decreases with an increase in $k^{*}$ for the case of an insulated 
Table 1

Normalized thermocapillary migration velocity of a spherical droplet parallel to a single plane wall computed from the exact boundary-collocation solution and the asymptotic method-of-reflection solution

\begin{tabular}{|c|c|c|c|c|}
\hline \multirow[t]{3}{*}{$a / b$} & \multicolumn{4}{|l|}{$U / U_{0}$} \\
\hline & \multicolumn{2}{|l|}{$k^{*}=\eta^{*}=0$} & \multicolumn{2}{|l|}{$k^{*}=\eta^{*}=10$} \\
\hline & Exact solution & Asymptotic solution & Exact solution & Asymptotic solution \\
\hline \multicolumn{5}{|c|}{ For an insulated plane wall } \\
\hline 0.2 & 0.99950 & 0.99950 & 0.99828 & 0.99827 \\
\hline 0.4 & 0.99577 & 0.99576 & 0.98648 & 0.98637 \\
\hline 0.6 & 0.98339 & 0.98377 & 0.95378 & 0.95238 \\
\hline 0.8 & 0.94340 & 0.95264 & 0.87831 & 0.87311 \\
\hline 0.9 & 0.88498 & 0.92330 & 0.79943 & 0.80170 \\
\hline 0.95 & 0.8186 & 0.90334 & 0.7269 & 0.75369 \\
\hline 0.99 & 0.6989 & 0.88419 & 0.6155 & 0.70780 \\
\hline 0.995 & 0.675 & & 0.594 & \\
\hline 0.999 & 0.654 & & 0.575 & \\
\hline \multicolumn{5}{|c|}{ For a plane wall prescribed with the far-field temperature profile } \\
\hline 0.2 & 0.99849 & 0.99849 & 0.99978 & 0.99978 \\
\hline 0.4 & 0.98764 & 0.98763 & 0.99871 & 0.99860 \\
\hline 0.6 & 0.95481 & 0.95531 & 0.99709 & 0.99552 \\
\hline 0.8 & 0.86997 & 0.88045 & 0.99327 & 0.98395 \\
\hline 0.9 & 0.77497 & 0.81557 & 0.98279 & 0.96846 \\
\hline 0.95 & 0.6879 & 0.77320 & 0.9633 & 0.95606 \\
\hline 0.99 & 0.5577 & 0.73348 & 0.9175 & 0.94302 \\
\hline 0.995 & 0.533 & & 0.909 & \\
\hline 0.999 & 0.513 & & 0.904 & \\
\hline
\end{tabular}

wall (the boundary condition (3c) is used), but increases with an increase in $k^{*}$ for the case of a plane wall prescribed with the far-field temperature distribution (the boundary condition (3e) is used), keeping the other factors $\left(\eta^{*}\right.$ and $\left.a / b\right)$ unchanged. These decrease and increase in the droplet mobility becomes more pronounced as $a / b$ increases. This behavior is expected knowing that the temperature gradients on the droplet surface near an insulated wall decrease as the relative conductivity $k^{*}$ increases and these gradients near a wall with the imposed far-field temperature gradient increase as $k^{*}$ increases. On the other hand, the wall-corrected normalized thermocapillary mobility of the droplet increases with an increase in $\eta^{*}$ for any given values of $k^{*}$ and $a / b$ in both cases of the plane wall, in agreement with the prediction from the method-ofreflection solution given by (A.11). As expected, when $k^{*}=1$, the two types of plane walls will result in the same effects on the thermocapillary motion of the droplet. In this particular case, the effect of thermal interaction between the droplet and the wall disappears, and the relative thermocapillary mobility of the droplet decreases monotonically with $a / b$ solely owing to the hydrodynamic resistance exerted by the presence of the wall.

Examination of the results shown in Figs. 2 and 3 reveals an interesting feature. For the case that the wall is an insulated plane under the situation of large $\eta^{*}$ and small $k^{*}$ (e.g., with $\eta^{*}=100$ and $k^{*}=0$ ), the thermocapillary mobility of the droplet decreases with an increase in $a / b$ as $a / b$ is small, but increases from a minimum with increasing $a / b$ as $a / b$ is sufficiently large. Under the 

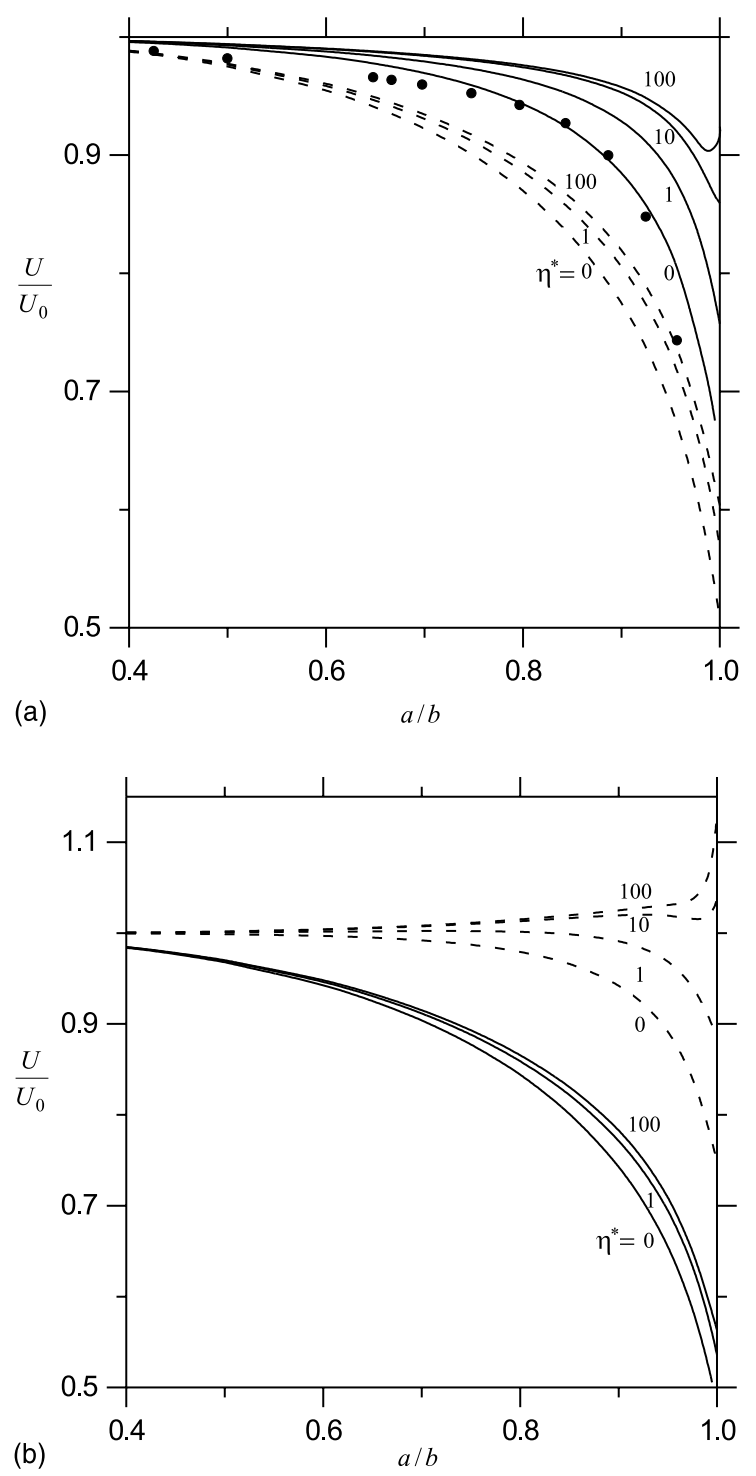

Fig. 2. Plots of the normalized thermocapillary mobility $U / U_{0}$ of a spherical droplet migrating parallel to a plane wall versus the separation parameter $a / b$ for various values of $\eta^{*}$ : (a) $k^{*}=0$; (b) $k^{*}=100$. The solid curves represent the case of an insulated wall, and the dashed curves denote the case of a wall on which the far-field temperature gradient is imposed. The points are the combined analytical-numerical results obtained by Meyyappan and Subramanian (1987) for the thermocapillary migration of a spherical bubble (with $k^{*}=\eta^{*}=0$ ) parallel to a plane wall prescribed with the far-field temperature distribution.

other situations (with small $\eta^{*}$ or large $k^{*}$ ), the thermocapillary mobility of the droplet near the insulated wall is a monotonically decreasing function of $a / b$. For the case that a linear temperature profile is prescribed on the plane wall which is consistent with the far-field distribution under the situation of large $\eta^{*}$ and large $k^{*}$ (e.g., with $\eta^{*}=100$ and $k^{*} \geqslant 10$ ), the thermocapillary 


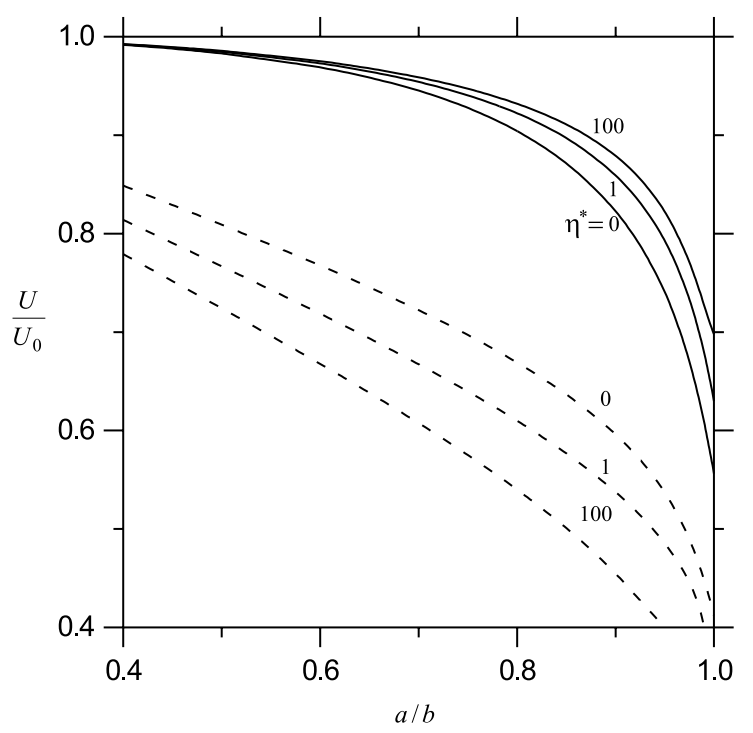

Fig. 3. Plots of the normalized thermocapillary mobility (solid curves, with $k^{*}=1$ ) and sedimenting mobility (dashed curves) of a spherical droplet migrating parallel to a plane wall versus the separation parameter $a / b$ for different values of $\eta^{*}$.

mobility of the droplet first goes through a minimum with the increase of $a / b$ from $a / b=0$ and then increases monotonically. When the gap between the droplet and the wall turns thin, the droplet can even move faster than it would at $a / b=0$. For example, at the values of $k^{*}=\eta^{*}=$ 100 and $a / b=0.999$, the thermocapillary velocity can be as much as $13 \%$ higher than the value with the wall being far away from the droplet. Under the situation of small $k^{*}$ or small $\eta^{*}$, the thermocapillary mobility of the droplet near the wall prescribed with the far-field temperature distribution becomes a monotonically decreasing function of $a / b$. Under some situations of intermediate values of $\eta^{*}$ (e.g., with $\eta^{*}=1-10$ and $k^{*}=100$ ), the droplet mobility as a function of $a / b$ can have two or three extremes (minima or maxima) in the whole range of $0<a / b<1$. This interesting feature that $U / U_{0}$ may not be a monotonic function of $a / b$ and can even be greater than unity is understandable because the wall effect of hydrodynamic resistance on the droplet is in the competition with the wall effect of thermal enhancement when a droplet with small $k^{*}$ is undergoing thermocapillary motion parallel to an insulated plate or when a droplet with large $k^{*}$ is moving near a lateral plate with the imposed far-field temperature gradient. A careful examination of the asymptotic formula for $U / U_{0}$ given by (A.11) shows a good agreement of the numerical outcome in Figs. 2 and 3 with the analytical solution.

Through the use of bipolar coordinates, Meyyappan and Subramanian (1987) obtained some semianalytical-seminumerical solutions for the normalized themocapillary velocity $U / U_{0}$ of a spherical gas bubble (with $k^{*}=\eta^{*}=0$ ) migrating parallel to a plane wall prescribed with the farfield temperaure distribution. These solutions are also presented in Fig. 2(a) for comparison. It can be seen that the bipolar-coordinate solution for $U / U_{0}$ of a bubble at a given value of $a / b$ is far greater than our corresponding collocation solution (illustrated by the lowest dashed curve in Fig. 2a). A detailed comparison given by Table 2 shows that our collocation solutions agree much 
Table 2

A comparison of the normalized thermocapillary migration velocity $U / U_{0}$ of a spherical bubble (with $k^{*}=\eta^{*}=0$ ) parallel to a single plane wall prescribed with the far-field temperature profile among the results obtained by Meyyappan and Subramanian (1987), our collocation solution, and the asymptotic reflection solution

\begin{tabular}{llll}
\hline$a / b$ & Meyyappan and Subramanian (1987) & Collocation solution & Asymptotic solution \\
\hline 0.95666 & 0.74314 & 0.67184 & 0.76697 \\
0.92498 & 0.84781 & 0.73728 & 0.79534 \\
0.88684 & 0.89967 & 0.79168 & 0.82551 \\
0.84353 & 0.92698 & 0.83627 & 0.85509 \\
0.79669 & 0.94236 & 0.87220 & 0.88221 \\
0.74772 & 0.95217 & 0.90082 & 0.90592 \\
0.69779 & 0.95953 & 0.92346 & 0.92592 \\
0.66667 & 0.96354 & 0.93503 & 0.93656 \\
0.64805 & 0.96581 & 0.94118 & 0.94231 \\
0.50000 & 0.98172 & 0.97511 & 0.97516 \\
0.42510 & 0.98808 & 0.98507 & 0.98507 \\
0.33333 & 0.99392 & 0.99293 & 0.99293 \\
0.26580 & 0.99681 & 0.99645 & 0.99645 \\
0.20000 & 0.99860 & 0.99849 & 0.99849 \\
0.16307 & 0.99922 & 0.99919 & 0.99919 \\
0.10000 & 0.99982 & 0.99981 & 0.99981 \\
0.06667 & 0.99995 & 0.99994 & 0.99994 \\
0.04000 & 0.99999 & 0.99999 & 0.99999 \\
\hline
\end{tabular}

better with the method-of-reflection solution given by (A.11) than the bipolar-coordinate solutions do for all values of $a / b$ less than 0.9 . It seems quite likely that these bipolar-coordinate solutions are in error. Note that the relative thermocapillary velocity of a gas bubble near a plane wall always decreases as the relative gap thickness decreases (or $a / b$ increases) no matter whether the wall is insulated or prescribed with the far-field temperature distribution.

For the creeping motion of a spherical droplet on which a constant body force $F \mathbf{e}_{x}$ (e.g., a gravitational field) is exerted parallel to an infinite plane wall, the exact result of the droplet velocity has recently been developed by using the boundary-collocation technique (Keh and Chen, 2001). A comparison of the boundary effects on the translation of the fluid sphere under gravity (in which $\left.U_{0}=(F / 6 \pi \eta a)\left(3 \eta^{*}+3\right) /\left(3 \eta^{*}+2\right)\right)$ and on the thermocapillary migration is given in Fig. 3. Obviously, the wall effect on thermocapillary motion is much weaker than that on a sedimenting or buoyantly rising droplet. Note that the wall effect on the droplet motion in gravitational field is stronger when the value of $\eta^{*}$ becomes larger, which is opposite to that which would occur if the droplet migrated near a plane wall due to thermocapillarity.

\subsection{Motion parallel to two plane walls}

Several of converged collocation solutions for the normalized velocity $U / U_{0}$ of a spherical droplet undergoing thermocapillary migration on the median plane between two parallel plane walls (with $c=b$ ) for various values of the parameters $k^{*}, \eta^{*}$, and $a / b$ are presented in Table 3 for both cases of insulated walls and walls prescribed with the far-field temperature distribution. The corresponding method-of-reflection soultions, given by (A.20) in Appendix A as a power series 
Table 3

Normalized velocity of a spherical droplet undergoing thermocapillary migration on the median plane between two parallel plane walls computed from the exact boundary-collocation solution and the asymptotic method-of-reflection solution

\begin{tabular}{|c|c|c|c|c|}
\hline \multirow[t]{3}{*}{$a / b$} & \multicolumn{4}{|l|}{$U / U_{0}$} \\
\hline & \multicolumn{2}{|l|}{$\overline{k^{*}}=\eta^{*}=0$} & \multicolumn{2}{|l|}{$k^{*}=\eta^{*}=10$} \\
\hline & Exact solution & Asymptotic solution & Exact solution & Asymptotic solution \\
\hline \multicolumn{5}{|c|}{ For insulated plane walls } \\
\hline 0.2 & 0.99786 & 0.99786 & 0.99496 & 0.99496 \\
\hline 0.4 & 0.98269 & 0.98270 & 0.96228 & 0.96267 \\
\hline 0.6 & 0.93986 & 0.94030 & 0.88483 & 0.89247 \\
\hline 0.8 & 0.84515 & 0.85239 & 0.75406 & 0.81225 \\
\hline 0.9 & 0.75426 & 0.78347 & 0.65752 & 0.79535 \\
\hline 0.95 & 0.6755 & 0.74091 & 0.5863 & 0.80043 \\
\hline 0.99 & 0.5571 & 0.70238 & 0.4915 & 0.81391 \\
\hline 0.995 & 0.535 & & 0.475 & \\
\hline 0.999 & 0.517 & & 0.461 & \\
\hline \multicolumn{5}{|c|}{ For plane walls prescribed with the far-field temperature profile } \\
\hline 0.2 & 0.99576 & 0.99576 & 0.99811 & 0.99811 \\
\hline 0.4 & 0.96621 & 0.96628 & 0.98692 & 0.98714 \\
\hline 0.6 & 0.88639 & 0.88817 & 0.96491 & 0.96893 \\
\hline 0.8 & 0.72600 & 0.74398 & 0.93828 & 0.96546 \\
\hline 0.9 & 0.59298 & 0.64494 & 0.92380 & 0.98347 \\
\hline 0.95 & 0.4954 & 0.58899 & 0.9092 & 1.00108 \\
\hline 0.99 & 0.3746 & 0.54141 & 0.8785 & 1.02050 \\
\hline 0.995 & 0.354 & & 0.875 & \\
\hline 0.999 & 0.338 & & 0.874 & \\
\hline
\end{tabular}

expansion in $\lambda(=a / b)$ correct to $\mathrm{O}\left(\lambda^{7}\right)$, are also listed in this table for comparison. Partly similar to the case of migration of a droplet parallel to a single plane wall considered in the previous subsection, the approximate analytical formula of (A.20) agrees very well with the exact results as long as $\lambda \leqslant 0.6$, but can have significant errors when $\lambda \geqslant 0.8$. The formula of (A.20) always overestimates the thermocapillary migration velocity of the droplet. A comparison between Table 3 for the case of a slit and Table 1 for the case of a single parallel plane indicates that the assumption that the boundary effect for two walls can be obtained by simple addition of single-wall effect leads to a smaller correction to thermocapillary motion as $a / b$ is small but can give a greater correction as $a / b$ becomes large.

In Fig. 4, the collocation results for the normalized thermocapillary mobility $U / U_{0}$ of a fluid sphere migrating on the median plane between two parallel plane walls are plotted as functions of $a / b$ for several values of $k^{*}$ and $\eta^{*}$. Analogous to the corresponding motion of a droplet parallel to a single plane wall, for specified values of $\eta^{*}$ and $a / b, U / U_{0}$ increases with an increase in $k^{*}$ for the case of walls with the imposed far-field temperature gradient and decreases with an increase in $k^{*}$ for the case of insulated walls. For either case of the plane walls, $U / U_{0}$ increases with an increase in $\eta^{*}$ for fixed values of $k^{*}$ and $a / b$. Again, for the case of insulated walls under the situation of large $\eta^{*}$ and small $k^{*}$, or for the case of walls prescribed with the far-field temperature distribution 


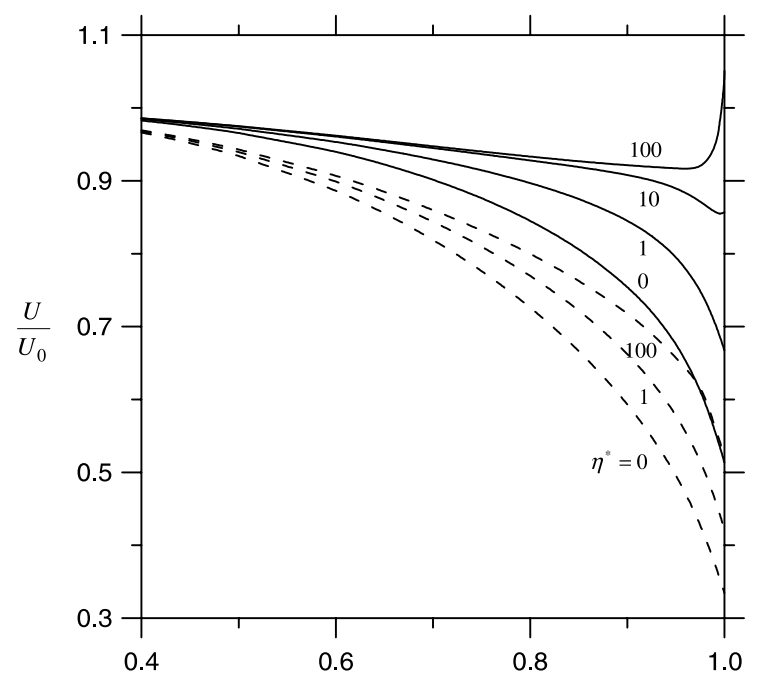

(a)

$a / b$

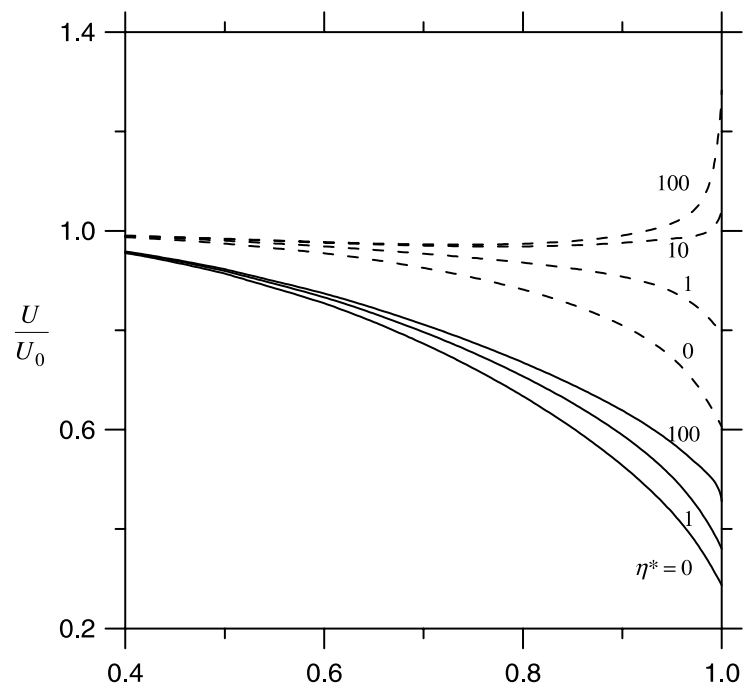

(b)

$a / b$

Fig. 4. Plots of the normalized thermocapillary mobility $U / U_{0}$ of a spherical droplet migrating on the median plane between two parallel plane walls (with $c=b$ ) versus the separation parameter $a / b$ for several values of $\eta^{*}$ : (a) $k^{*}=0$; (b) $k^{*}=100$. The solid curves represent the case of insulated walls, and the dashed curves denote the case of walls prescribed with the far-field temperature distribution.

under the situation of large $\eta^{*}$ and large $k^{*}$, the thermocapillary mobility of the droplet first goes through a minimum with the increase of $a / b$ from $a / b=0$ and then increases monotonically, and the droplet can even move faster than it would at $a / b=0$. This result indicates that the effect of thermal enhancement, rather than that of hydrodynamic resistance, can be overriding when the droplet-wall gap thickness is small. An examination of the asymptotic formula for $U / U_{0}$ in (A.20) also shows a good agreement of the trend in Fig. 4 with the analytical solution. 
A careful comparison of the curves in Fig. 4 for the case of a slit with the corresponding curves in Fig. 2 for the case of a single wall reveals an interesting feature of the boundary effect on thermocapillary migration of a fluid sphere. The presence of a second, identical, lateral plane wall, even at a symmetric position with respect to the sphere against the first, does not always enhance the wall effect on the thermocapillary droplet induced by the first plate only. In fact, the wall effects on the droplet mobility for the two bounded cases can lead to opposite results relative to its isolated value. For example, the thermocapillary mobility of a droplet parallel to a single plate prescribed with the far-field temperature distribution at values $k^{*}=100, \eta^{*}=10$, and $a / b=0.8$ is greater than that in an unbounded fluid by about $1.5 \%$, while the mobility of the droplet undergoing thermocapillary migration on the central plane of a slit under identical conditions is about $2.5 \%$ smaller than its isolated value. These results reflect again the fact that the lateral wall can affect the thermal driving force and the viscous drag force on a fluid droplet in opposite directions. Each force is increased in its own direction, but to a different degree, for the case of thermocapillary motion of a droplet in a slit relative to that for the case of migration parallel to a single plate. Thus, the net effect composed of these two opposite forces for the slit case is not necessarily to enhance that for the case of a single wall.

Fig. 5 shows the collocation results for the normalized mobility $U / U_{0}$ of a fluid sphere undergoing thermocapillary motion parallel to two plane walls at various positions between them for a general case (with $k^{*}=\eta^{*}=1$ ). The dashed curves (with $a / b=$ constant) illustrate the effect of the position of the second wall (at $z=c$ ) on the droplet mobility for various values of the relative sphere-to-first-wall spacing $b / a$. The solid curves (with $2 a /(b+c)=$ constant) indicate the variation of the droplet mobility as a function of the sphere position at various values of the relative wall-to-wall spacing $(b+c) / 2 a$. It can be seen that the net wall effect is to reduce the thermo-

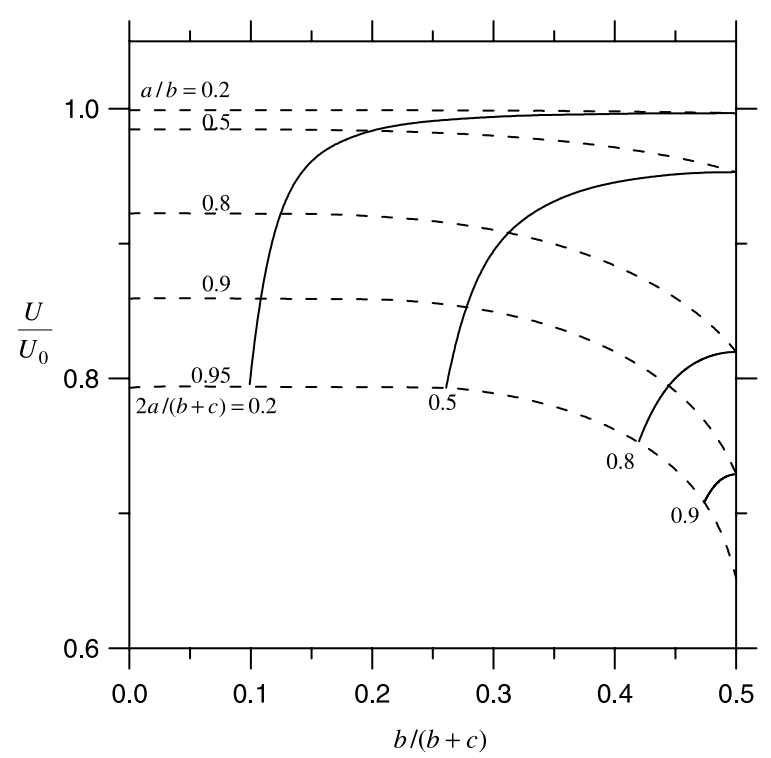

Fig. 5. Plots of the normalized thermocapillary mobility $U / U_{0}$ of a spherical droplet migrating parallel to two plane walls versus the ratio $b /(b+c)$ for the case of $k^{*}=\eta^{*}=1$ with $a / b$ and $2 a /(b+c)$ as parameters. 
capillary mobility of the droplet. At a given value of $2 a /(b+c)$, the droplet experiences a minimum viscous drag force and has a greatest mobility when it is located midway between the two walls (with $c=b$ ). The hydrodynamic drag increases and the droplet mobility decreases as the droplet approaches either of the walls (or the ratio $b /(b+c)$ decreases). At a specified value of $a / b$ for the thermocapillary droplet near a first lateral wall, the presence of a second plate is to further reduce the relative velocity of the droplet, and the degree of this reduction increases monotonically with a decrease in the relative distance between the droplet and the second plate (or with an increase in $b /(b+c))$.

The collocation solution for the problem of sedimentation of a fluid sphere parallel to two plane walls at an arbitrary position between them was also obtained (Keh and Chen, 2001). Comparing that solution with the present result, we still find that the wall effect on thermocapillary migration in general is much weaker than that on sedimentation. Opposite to the cases of thermocapillary migration illustrated in Fig. 4, the retardation effect on the sedimenting droplet in a slit is stronger for a greater value of $\eta^{*}$.

\section{Conclusions}

In this work, the exact numerical solutions and approximate analytical solutions for the steady thermocapillary motion of a fluid sphere parallel to two infinite plane walls at an arbitrary position between them have been obtained by using the boundary-collocation technique and the method of reflections, respectively. Both the cases of insulated walls and of walls with the imposed far-field temperature gradient were examined in the limit of vanishingly small Reynolds and Peclet numbers. It has been found that the boundary effect on thermocapillary motion of a fluid particle is quite complicated. The thermocapillary mobility of a droplet near a plane wall is generally, but not necessarily, a monotonic decreasing function of the separation parameter $a / b$. When the relative viscosity of the droplet $\eta^{*}$ is large and the value of $a / b$ is close to unity, the wall effect can speed up or slow down the droplet velocity relative to its isolated value depending on the value of the relative thermal conductivity of the droplet $k^{*}$ and the thermal boundary condition at the wall. This behavior reflects the competition between the weak hydrodynamic retardation exerted by the neighboring walls on the droplet migration and the possible, relatively strong thermocapillary enhancement due to the thermal interaction between the droplet and the lateral walls.

\section{Acknowledgements}

This research was partly supported by the National Science Council of the Republic of China.

\section{Appendix A. Analysis of the thermocapillary migration of a droplet parallel to plane walls by a method of reflections}

In this appendix, we analyze the steady thermocapillary motion of a fluid sphere with relative thermal conductivity $k^{*}$ and relative viscosity $\eta^{*}$ either parallel to an infinite flat wall $(c \rightarrow \infty)$ or 
on the median plane between two parallel plates $(c=b)$, as shown in Fig. 1, by a method of reflections. The effect of the walls on the droplet velocity is sought in expansions of $\lambda$, which equals $a / b$, the ratio of the droplet radius to the distance between the walls and the center of the droplet.

\section{A.1. Motion parallel to an infinite plane wall}

For the problem of thermocapillary motion parallel to an insulated plane wall, the governing equations in (2a) and (10) must be solved by satisfying the boundary conditions (3a)-(3d) and (12a)-(12e) with $c \rightarrow \infty$. The method-of-reflection solution consists of the following series, whose terms depend on increasing powers of $\lambda$ :

$$
\begin{aligned}
& T=T_{0}+E_{\infty} x+T_{\mathrm{p}}^{(1)}+T_{\mathrm{w}}^{(1)}+T_{\mathrm{p}}^{(2)}+T_{\mathrm{w}}^{(2)}+\cdots, \\
& \mathbf{v}=\mathbf{v}_{\mathrm{p}}^{(1)}+\mathbf{v}_{\mathrm{w}}^{(1)}+\mathbf{v}_{\mathrm{p}}^{(2)}+\mathbf{v}_{\mathrm{w}}^{(2)}+\cdots,
\end{aligned}
$$

where subscripts $\mathrm{w}$ and $\mathrm{p}$ represent the reflections from wall and droplet, respectively, and the superscript (i) denotes the $i$ th reflection from that surface. In these series, all the expansion sets of the corresponding temperature and velocity for the external fluid must satisfy (2a) and (10). The advantage of this method is that it is necessary to consider boundary conditions associated with only one surface at a time.

According to (A.1), the thermocapillary migration velocity of the droplet can also be expressed in the series form,

$$
\mathbf{U}=U_{0} \mathbf{e}_{x}+\mathbf{U}^{(1)}+\mathbf{U}^{(2)}+\cdots,
$$

In this expression, $U_{0}$ is the thermocapillary velocity of an identical droplet suspended freely in the continuous phase far from the wall given by (1); $\mathbf{U}^{(i)}$ is related to $\nabla T_{\mathrm{w}}^{(i)}$ and $\mathrm{v}_{\mathrm{w}}^{(i)}$ by (Anderson, 1985; Chen and Keh, 1990)

$$
\mathbf{U}^{(i)}=A \frac{a}{\eta}\left(-\frac{\partial \gamma}{\partial T}\right)\left[\nabla T_{\mathrm{w}}^{(i)}\right]_{0}+\left[\mathbf{v}_{\mathrm{w}}^{(i)}\right]_{0}+C \frac{a^{2}}{6}\left[\nabla^{2} \mathbf{v}_{\mathrm{w}}^{(i)}\right]_{0} .
$$

Here, $A=2\left(2+k^{*}\right)^{-1}\left(2+3 \eta^{*}\right)^{-1}, C=3 \eta^{*}\left(2+3 \eta^{*}\right)^{-1}$, and the subscript 0 to variables inside brackets denotes evaluation at the position of the droplet center. Note that $0 \leqslant A \leqslant 1 / 2$ and $0 \leqslant C \leqslant 1$. The last two terms in (A.3) represent the Faxen law for the isothermal motion of a force-free fluid sphere (Hetsroni and Haber, 1970).

The solution for the first reflected fields from the droplet is

$$
\begin{aligned}
T_{\mathrm{p}}^{(1)} & =G E_{\infty} a^{3} r^{-2} \sin \theta \cos \phi, \\
\mathbf{v}_{\mathrm{p}}^{(1)} & =\frac{1}{2} U_{0} a^{3} r^{-3}\left(2 \sin \theta \cos \phi \mathbf{e}_{r}-\cos \theta \cos \phi \mathbf{e}_{\theta}+\sin \phi \mathbf{e}_{\phi}\right),
\end{aligned}
$$

where $G=\left(1-k^{*}\right)\left(2+k^{*}\right)^{-1}$. Obviously, $-1 \leqslant G \leqslant 1 / 2$, with the upper and lower bounds occurring at the limits $k^{*}=0$ and $k^{*} \rightarrow \infty$, respectively. The velocity distribution shown in (A.4b) is identical to the irrotational flow surrounding a rigid sphere moving with velocity $U_{0} \mathbf{e}_{x}$. 
The boundary conditions for the $i$ th reflected fields from the wall are derived from $(3 \mathrm{c}),(3 \mathrm{~d})$, (12d) and (12e),

$$
\begin{aligned}
& z=-b: \quad \frac{\partial T_{\mathrm{w}}^{(i)}}{\partial z}=-\frac{\partial T_{\mathrm{p}}^{(i)}}{\partial z}, \\
& z=-b: \quad \mathbf{v}_{\mathrm{w}}^{(i)}=-\mathbf{v}_{\mathrm{p}}^{(i)}, \\
& r \rightarrow \infty, z>-b: \quad T_{\mathrm{w}}^{(i)} \rightarrow 0, \\
& r \rightarrow \infty, z>-b: \quad \mathbf{v}_{\mathrm{w}}^{(i)} \rightarrow \mathbf{0} .
\end{aligned}
$$

The solution of $T_{\mathrm{w}}^{(1)}$ is obtained by applying complex Fourier transforms on $x$ and $y$ in (2a), (A.5a) and (A.5c), with the result

$$
T_{\mathrm{w}}^{(1)}=G E_{\infty} a^{3} x\left[x^{2}+y^{2}+(z+2 b)^{2}\right]^{-3 / 2} .
$$

This reflected temperature field may be interpreted as arising from the reflection of the imposed field $E_{\infty} \mathbf{e}_{x}$ from a fictitious droplet identical to the actual droplet, its location being at the mirrorimage position of the actual droplet with respect to the plane $z=-b$ (i.e. at $x=0, y=0$, $z=-2 b$ ). The solution of $\mathbf{v}_{\mathrm{w}}^{(1)}$ can be found by fitting the boundary conditions (A.5b) and (A.5d) with the general solution to (10) established by Faxen (Happel and Brenner, 1983), which results in

$$
\begin{aligned}
\mathbf{v}_{\mathrm{w}}^{(1)}= & \frac{U_{0} a^{3}}{4 \pi} \int_{-\infty}^{\infty} \int_{-\infty}^{\infty} \mathrm{e}^{\mathrm{i}(\alpha x+\beta y)-\kappa(z+2 b)}\left\{-[2 \kappa(z+b)+1] \mathrm{i} \alpha \mathbf{e}_{z}\right. \\
& \left.-[2 \kappa(z+b)-1]\left(\frac{\alpha^{2}}{\kappa} \mathbf{e}_{x}+\frac{\alpha \beta}{\kappa} \mathbf{e}_{y}\right)\right\} \mathrm{d} \alpha \mathrm{d} \beta,
\end{aligned}
$$

where $\kappa=\left(\alpha^{2}+\beta^{2}\right)^{1 / 2}$, and $\mathrm{i}=\sqrt{-1}$.

The contributions of $T_{\mathrm{w}}^{(1)}$ and $\mathbf{v}_{\mathrm{w}}^{(1)}$ to the droplet velocity are determined by using (A.3),

$$
\begin{aligned}
& \mathbf{U}_{\mathrm{t}}^{(1)}=A \frac{a}{\eta}\left(-\frac{\partial \gamma}{\partial T}\right)\left[\nabla T_{\mathrm{w}}^{(1)}\right]_{r=0}=\frac{1}{8} G \lambda^{3} U_{0} \mathbf{e}_{x}, \\
& \mathbf{U}_{\mathrm{h}}^{(1)}=\left[\mathbf{v}_{\mathrm{w}}^{(1)}+C \frac{a^{2}}{6} \nabla^{2} \mathbf{v}_{\mathrm{w}}^{(1)}\right]_{r=0}=-\frac{1}{8}\left(\lambda^{3}-C \lambda^{5}\right) U_{0} \mathbf{e}_{x}, \\
& \mathbf{U}^{(1)}=\mathbf{U}_{\mathrm{t}}^{(1)}+\mathbf{U}_{\mathrm{h}}^{(1)}=\frac{1}{8}\left[-(1-G) \lambda^{3}+C \lambda^{5}\right] U_{0} \mathbf{e}_{x} .
\end{aligned}
$$

Eq. (A.7a) shows that the reflected temperature field from the insulated wall can increase (if $G>0$ or $k^{*}<1$ ) or decrease (if $G<0$ or $k^{*}>1$ ) the velocity of the thermocapillary droplet, while (A.7b) indicates that the reflected velocity field is to decrease this velocity; the net effect of the reflected fields is expressed by $(\mathrm{A} .7 \mathrm{c})$, which can enhance or retard the movement of the droplet, depending on the combination of the values of $G\left(\right.$ or $\left.k^{*}\right), \eta^{*}$, and $\lambda$. When $G=0$ (or $k^{*}=1$ ), the reflected temperature field makes no contribution to the thermocapillary migration velocity. Eqs. (A.7a)(A.7c) indicate that the wall correction to the velocity of the thermocapillary droplet is $\mathrm{O}\left(\lambda^{3}\right)$, which is weaker than that obtained for the corresponding sedimentation problem, in which the leading boundary effect is $\mathrm{O}(\lambda)$. Note that the wall effect on thermocapillary motion involving the 
viscosity parameter $\eta^{*}$ appears starting from $\mathrm{O}\left(\lambda^{5}\right)$, and the normalized droplet velocity increases with an increase in $\eta^{*}$. The necessary conditions for the wall enhancement on the thermocapillary motion to occur are a small value of $k^{*}$, a large value of $\eta^{*}$, and a value of $\lambda$ close to unity such that the relation $C \lambda^{5}>(1-G) \lambda^{3}$ is warranted.

The solution for the second reflected fields from the droplet is

$$
\begin{aligned}
T_{\mathrm{p}}^{(2)}= & \frac{1}{8} E_{\infty}\left[G^{2} \lambda^{3} a^{3} r^{-2} \sin \theta \cos \phi+G H \lambda^{4} a^{4} r^{-3} \cos \theta \sin \theta \cos \phi+\mathrm{O}\left(\lambda^{5} a^{5}\right)\right], \\
\mathbf{v}_{\mathrm{p}}^{(2)}= & \frac{1}{32} U_{0}\left[2 G \lambda^{3} a^{3} r^{-3}\left(2 \sin \theta \cos \phi \mathbf{e}_{r}-\cos \theta \cos \phi \mathbf{e}_{\theta}+\sin \phi \mathbf{e}_{\phi}\right)\right. \\
& \left.-3\left(D-2 G \frac{B}{A}\right) \lambda^{4} a^{2} r^{-2} \cos \theta \sin \theta \cos \phi \mathbf{e}_{r}+\mathrm{O}\left(\lambda^{4} a^{4}, \lambda^{5} a^{3}\right)\right] .
\end{aligned}
$$

Here, $H=3\left(1-k^{*}\right)\left(3+2 k^{*}\right)^{-1}, B=3\left(3+2 k^{*}\right)^{-1}\left(1+\eta^{*}\right)^{-1}$, and $D=3\left(2+5 \eta^{*}\right)\left(1+\eta^{*}\right)^{-1}$.

The boundary conditions for the second reflected fields from the wall are obtained by substituting the results of $T_{\mathrm{p}}^{(2)}$ and $\mathbf{v}_{\mathrm{p}}^{(2)}$ into (A.5a)-(A.5d), with which (2a) and (10) can be solved as before to yield

$$
\begin{aligned}
& {\left[\nabla T_{\mathrm{w}}^{(2)}\right]_{r=0}=\left[\frac{1}{64} G^{2} \lambda^{6}+\mathrm{O}\left(\lambda^{8}\right)\right] E_{\infty} \mathbf{e}_{x},} \\
& {\left[\mathbf{v}_{\mathrm{w}}^{(2)}\right]_{r=0}=\left\{-\frac{1}{256}\left[3 D-2\left(3 \frac{B}{A}-2\right) G\right] \lambda^{6}+\mathrm{O}\left(\lambda^{8}\right)\right\} U_{0} \mathbf{e}_{x} .}
\end{aligned}
$$

The contribution of the second reflected fields to the droplet velocity is obtained by putting $T_{\mathrm{w}}^{(2)}$ and $\mathbf{v}_{\mathrm{w}}^{(2)}$ into (A.3), which gives

$$
\mathbf{U}^{(2)}=\left\{-\frac{1}{256}\left[3 D-2\left(3 \frac{B}{A}-2\right) G-4 G^{2}\right] \lambda^{6}+\mathrm{O}\left(\lambda^{8}\right)\right\} U_{0} \mathbf{e}_{x} .
$$

The errors for $\mathbf{U}^{(2)}$ is $\mathrm{O}\left(\lambda^{8}\right)$, because the $\mathrm{O}\left(\lambda^{7}\right)$ terms in the expansions of $\nabla T_{\mathrm{w}}^{(2)}$ and $\mathbf{v}_{\mathrm{w}}^{(2)}$ vanish at the center of the droplet.

Obviously, $\mathbf{U}^{(3)}$ will be $\mathrm{O}\left(\lambda^{9}\right)$. With the substitution of (A.7c) and (A.10) into (A.2), the droplet velocity can be expressed as $\mathbf{U}=U \mathbf{e}_{x}$ with

$$
U=U_{0}\left\{1-\frac{1}{8}(1-G) \lambda^{3}+\frac{C}{8} \lambda^{5}-\frac{1}{256}\left[3 D-2\left(3 \frac{B}{A}-2\right) G-4 G^{2}\right] \lambda^{6}+\mathrm{O}\left(\lambda^{8}\right)\right\} .
$$

The droplet migrates along the imposed temperature gradient at a rate that can increase or decrease as the droplet approaches the wall. Owing to the neglect of the inertial effect, the wall does not deflect the direction of thermocapillary migration.

For the case that a linear temperature profile is prescribed on the plane wall which is consistent with the far-field distribution, namely, the boundary condition (3c) is replaced by (3e), the series expansions (A.1) and (A.2), the solution of $T_{\mathrm{p}}^{(1)}$ and $\mathbf{v}_{\mathrm{p}}^{(1)}$ in (A.4a) and (A.4b), and the boundary conditions for $T_{\mathrm{w}}^{(i)}$ and $\mathbf{v}_{\mathrm{w}}^{(i)}$ in (A.5b)-(A.5d) are still valid, while (A.5a) becomes

$$
z=-b: \quad T_{\mathrm{w}}^{(i)}=-T_{\mathrm{p}}^{(i)} .
$$


With this change, it can be shown that the results of the reflected fields and of the droplet velocity are also obtained from (A.6a)-(A.11) by replacing $G$ by $-G$. Thus, contrary to the effect of an insulated plane wall, the reflected temperature field from a parallel wall with the imposed far-field temperature gradient reduces the droplet velocity if $G>0$ or $k^{*}<1$ and enhances this velocity if $G<0$ or $k^{*}>1$. When $G=0$ or $k^{*}=1$, the two types of plane wall will produce the same effects on the thermocapillary motion of the droplet. Under the conditions that the values of $k^{*}, \eta^{*}$ and $\lambda$ are sufficiently large such that $C \lambda^{5}>(1+G) \lambda^{3}$, the net effect of a lateral plane wall prescribed with the far-field temperature distribution can enhance the thermocapillary migration of a droplet.

\section{A.2. Motion on the median plane between two parallel flat walls}

For the problem of thermocapillary motion on the median plane between two insulated parallel plates, the boundary conditions corresponding to governing equations (2a) and (10) are given by (3a)-(3d) and (12a)-(12e) with $c=b$. With $\lambda=a / b \ll 1$, the series expansions of the temperature, fluid velocity, and droplet velocity given by (A.1)-(A.4b) remain valid here. From (3c), (3d), (12d) and $(12 \mathrm{e})$, the boundary conditions for $T_{\mathrm{w}}^{(i)}$ and $\mathbf{v}_{\mathrm{w}}^{(i)}$ are found to be

$$
\begin{aligned}
& |z|=b: \quad \frac{\partial T_{\mathrm{w}}^{(i)}}{\partial z}=-\frac{\partial T_{\mathrm{p}}^{(i)}}{\partial z}, \\
& |z|=b: \quad \mathbf{v}_{\mathrm{w}}^{(i)}=-\mathbf{v}_{\mathrm{p}}^{(i)} ; \\
& r \rightarrow \infty,|z| \leqslant b: \quad T_{\mathrm{w}}^{(i)} \rightarrow 0 \\
& r \rightarrow \infty,|z| \leqslant b: \quad \mathbf{v}_{\mathrm{w}}^{(i)} \rightarrow \mathbf{0} .
\end{aligned}
$$

The first wall-reflected fields can be solved by the same method as used for a single lateral plate in the previous subsection, with the results

$$
\begin{aligned}
T_{\mathrm{w}}^{(1)}= & -\frac{G E_{\infty} a^{3}}{2 \pi} \int_{-\infty}^{\infty} \int_{-\infty}^{\infty} \frac{\mathrm{i} \alpha}{\kappa} \mathrm{e}^{\mathrm{i}(\alpha x+\beta y)-\kappa b} \frac{\cosh (\kappa z)}{\sinh (\kappa b)} \mathrm{d} \alpha \mathrm{d} \beta, \\
\mathbf{v}_{\mathrm{w}}^{(1)}= & \frac{U_{0} a^{3}}{2 \pi} \int_{-\infty}^{\infty} \int_{-\infty}^{\infty} \frac{1}{\sinh (2 \kappa b)-2 \kappa b} \\
& \times \mathrm{e}^{\mathrm{i}(\alpha x+\beta y)}\left\{[\sinh (\kappa z)-\kappa z \cosh (\kappa z)+g \sinh (\kappa z)] \mathrm{i} \alpha \mathbf{e}_{z}\right. \\
& \left.+[\kappa z \sinh (\kappa z)-g \cosh (\kappa z)]\left(\frac{\alpha^{2}}{\kappa} \mathbf{e}_{x}+\frac{\alpha \beta}{\kappa} \mathbf{e}_{y}\right)\right\} \mathrm{d} \alpha \mathrm{d} \beta,
\end{aligned}
$$

where $\kappa=\left(\alpha^{2}+\beta^{2}\right)^{1 / 2}$ and $g=\kappa b-\mathrm{e}^{-\kappa b} \sinh (\kappa b)$. The contributions of $T_{\mathrm{w}}^{(1)}$ and $\mathbf{v}_{\mathrm{w}}^{(1)}$ to the droplet velocity are determined using (A.3), which lead to a result similar to (A.7a)-(A.7c), 


$$
\begin{aligned}
& \mathbf{U}_{\mathrm{t}}^{(1)}=d_{1} G \lambda^{3} U_{0} \mathbf{e}_{x}, \\
& \mathbf{U}_{\mathrm{h}}^{(1)}=-\left(d_{2} \lambda^{3}-d_{3} C \lambda^{5}\right) U_{0} \mathbf{e}_{x}, \\
& \mathbf{U}^{(1)}=\mathbf{U}_{\mathrm{t}}^{(1)}+\mathbf{U}_{\mathrm{h}}^{(1)}=\left[-\left(d_{2}-d_{1} G\right) \lambda^{3}+d_{3} C \lambda^{5}\right] U_{0} \mathbf{e}_{x},
\end{aligned}
$$

where

$$
\begin{aligned}
& d_{1}=\int_{0}^{\infty} \frac{\rho^{2}}{\mathrm{e}^{2 \rho}-1} \mathrm{~d} \rho=0.300514 \\
& d_{2}=\frac{1}{2} \int_{0}^{\infty} \frac{\rho^{2}\left(\rho-\mathrm{e}^{-\rho} \sinh \rho\right)}{\sinh (2 \rho)-2 \rho} \mathrm{d} \rho=0.417956 \\
& d_{3}=\frac{1}{6} \int_{0}^{\infty} \frac{\rho^{4}}{\sinh (2 \rho)-2 \rho} \mathrm{d} \rho=0.338324
\end{aligned}
$$

Analogous to the previous case, the results of the second reflections can be obtained as

$$
\begin{aligned}
& T_{\mathrm{p}}^{(2)}=E_{\infty}\left[d_{1} G^{2} \lambda^{3} a^{3} r^{-2} \sin \theta \cos \phi+\mathrm{O}\left(\lambda^{5} a^{5}\right)\right], \\
& \mathbf{v}_{\mathrm{p}}^{(2)}=\frac{U_{0}}{2}\left[d_{1} G \lambda^{3} a^{3} r^{-3}\left(2 \sin \theta \cos \phi \mathbf{e}_{r}-\cos \theta \cos \phi \mathbf{e}_{\theta}+\sin \phi \mathbf{e}_{\phi}\right)+\mathrm{O}\left(\lambda^{5} a^{3}\right)\right], \\
& {\left[\nabla T_{\mathrm{w}}^{(2)}\right]_{r=0}=\left[d_{1}^{2} G^{2} \lambda^{6}+\mathrm{O}\left(\lambda^{8}\right)\right] E_{\infty} \mathbf{e}_{x},} \\
& {\left[\mathbf{v}_{\mathrm{w}}^{(2)}\right]_{r=0}=\left[-d_{1} d_{2} G \lambda^{6}+\mathrm{O}\left(\lambda^{8}\right)\right] U_{0} \mathbf{e}_{x},}
\end{aligned}
$$

and

$$
\mathbf{U}^{(2)}=\left[-\left(d_{1} d_{2} G-d_{1}^{2} G^{2}\right) \lambda^{6}+\mathrm{O}\left(\lambda^{8}\right)\right] U_{0} \mathbf{e}_{x} .
$$

Note that the $\lambda^{4} a^{2}$ and $\lambda^{4} a^{4}$ terms in the expressions for $T_{\mathrm{p}}^{(2)}$ and $\mathbf{v}_{\mathrm{p}}^{(2)}$ vanish. With the combination of (A.2), (A.15), and (A.19), the droplet velocity can be expressed as $\mathbf{U}=U \mathbf{e}_{x}$ with

$$
U=U_{0}\left[1-\left(d_{2}-d_{1} G\right) \lambda^{3}+d_{3} C \lambda^{5}-\left(d_{1} d_{2} G-d_{1}^{2} G^{2}\right) \lambda^{6}+\mathrm{O}\left(\lambda^{8}\right)\right] .
$$

For the case that the droplet is undergoing thermocapillary migration on the median plane between two parallel plates on which a linear temperature profile consistent with the far-field distribution is imposed, (3c) should be replaced by (3e). In this case, (A.1)-(A.4b) and (A.13b)(A.13d) are still applicable, while (A.13a) becomes

$$
|z|=b: \quad T_{\mathrm{w}}^{(i)}=-T_{\mathrm{p}}^{(i)} .
$$

With this change, it can be shown that the results of the reflected fields and of the droplet velocity are also obtained from (A.14)-(A.20) by replacing $G$ and $d_{1}$ by $-G$ and $\bar{d}_{1}$, respectively, where

$$
\bar{d}_{1}=\int_{0}^{\infty} \frac{\rho^{2}}{\mathrm{e}^{2 \rho}+1} \mathrm{~d} \rho=0.225386 .
$$

Comparing (A.20) for the slit case with (A.11) for the case of a single parallel plane, one can find that the wall effects on the thermocapillary motion of a droplet in the two cases are qualitatively similar. However, the assumption that the result of the boundary effect for two walls can be obtained by simple addition of the single-wall effect generally gives a smaller correction to 
thermocapillary motion, while for the corresponding sedimentation problem this approximation overestimates the wall correction (Happel and Brenner, 1983).

\section{Appendix B. Definitions of some functions in Section 2}

The functions $\delta_{n}^{(i)}$ with $i=1,2$, and 3 in (8), (9), (23e), and (23f) are defined by

$$
\begin{aligned}
\delta_{n}^{(1)}(r, \mu)= & r^{-n-1} P_{n}^{1}(\mu)-(-n)^{m} \int_{0}^{\infty} \frac{\kappa^{1-m} J_{1}(\kappa \rho)}{\sinh \tau}\left[c^{2} V_{n+m}(c)(\sinh \sigma)^{1-m}(\cos \sigma)^{m}\right. \\
& \left.-b^{2} V_{n+m}(-b)(\sinh \omega)^{1-m}(\cosh \omega)^{m}\right] \mathrm{d} \kappa \\
\delta_{n}^{(2)}(r, \mu)=- & (n+1) r^{-n-2} P_{n}^{1}(\mu)-(-n)^{m} \int_{0}^{\infty} \frac{\kappa^{2-m}}{\sinh \tau}\left\{c ^ { 2 } V _ { n + m } ( c ) \left[\mu J_{1}(\kappa \rho)(\cosh \sigma)^{1-m}(\sinh \sigma)^{m}\right.\right. \\
& \left.+\left(1-\mu^{2}\right)^{1 / 2} J_{1}^{\prime}(\kappa \rho)(\sinh \sigma)^{1-m}(\cosh \sigma)^{m}\right]-b^{2} V_{n+m}(-b)\left[\mu J_{1}(\kappa \rho)(\cosh \omega)^{1-m}(\sinh \omega)^{m}\right. \\
& \left.\left.+\left(1-\mu^{2}\right)^{1 / 2} J_{1}^{\prime}(\kappa \rho)(\sinh \omega)^{1-m}(\cosh \omega)^{m}\right]\right\} \mathrm{d} \kappa, \\
\delta_{n}^{(3)}(r, \mu)=- & r^{-n-1} \frac{d P_{n}^{1}(\mu)}{d \mu}\left(1-\mu^{2}\right)^{1 / 2}+(-n)^{m} r \int_{0}^{\infty} \frac{\kappa^{2-m}}{\sinh \tau}\left\{c^{2} V_{n+m}(c)\right. \\
& \times\left[\left(1-\mu^{2}\right)^{1 / 2} J_{1}(\kappa \rho)(\cosh \sigma)^{1-m}(\sinh \sigma)^{m}-\mu J_{1}^{\prime}(\kappa \rho)(\sinh \sigma)^{1-m}(\cosh \sigma)^{m}\right] \\
& -b^{2} V_{n+m}(-b)\left[\left(1-\mu^{2}\right)^{1 / 2} J_{1}(\kappa \rho)(\cosh \omega)^{1-m}(\sinh \omega)^{m}\right. \\
& \left.\left.\quad-\mu J_{1}^{\prime}(\kappa \rho)(\sinh \omega)^{1-m}(\cosh \omega)^{m}\right]\right\} \mathrm{d} \kappa .
\end{aligned}
$$

Here,

$$
\begin{aligned}
& V_{n}\left(z_{i}\right)=\frac{(2 / \pi)^{1 / 2}}{z_{i}^{n+1}} \sum_{q=0}^{[n / 2]} \frac{\left(\kappa\left|z_{i}\right|\right)^{n-q-(1 / 2)}}{(-2)^{q} q !(n-2 q-1) !} K_{n-q-(3 / 2)}\left(\kappa\left|z_{i}\right|\right), \\
& \sigma=\kappa(z+b), \quad \omega=\kappa(z-c), \quad \tau=\kappa(b+c),
\end{aligned}
$$

$J_{1}$ is the Bessel function of the first kind of order one and the prime on it denotes differentiation with respect to its argument, $K_{v}$ is the modified Bessel function of the second kind of order $v$, and the square bracket $[v]$ denotes the largest integer which is less than or equal to $v$. In (B.1a) and (B.1b) $m=0$ if (3e) is used for the boundary condition of the temperature field at the plane walls and $m=1$ if (3c) is used.The starred $A_{n}, B_{n}$, and $C_{n}$ functions in (23a)-(23f) are defined by

$$
\begin{aligned}
& A_{n}^{*}(r, \mu, \phi)=\left(1-\mu^{2}\right)^{1 / 2}\left(A_{n}^{\prime}+\alpha_{n}^{\prime}\right) \cos \phi+\left(1-\mu^{2}\right)^{1 / 2}\left(A_{n}^{\prime \prime}+\alpha_{n}^{\prime \prime}\right) \sin \phi+\mu\left(A_{n}^{\prime \prime \prime}+\alpha_{n}^{\prime \prime \prime}\right), \\
& B_{n}^{*}(r, \mu, \phi)=\left(1-\mu^{2}\right)^{1 / 2}\left(B_{n}^{\prime}+\beta_{n}^{\prime}\right) \cos \phi+\left(1-\mu^{2}\right)^{1 / 2}\left(B_{n}^{\prime \prime}+\beta_{n}^{\prime \prime}\right) \sin \phi+\mu\left(B_{n}^{\prime \prime \prime}+\beta_{n}^{\prime \prime \prime}\right), \\
& C_{n}^{*}(r, \mu, \phi)=\left(1-\mu^{2}\right)^{1 / 2}\left(C_{n}^{\prime}+\gamma_{n}^{\prime}\right) \cos \phi+\left(1-\mu^{2}\right)^{1 / 2}\left(C_{n}^{\prime \prime}+\gamma_{n}^{\prime \prime}\right) \sin \phi+\mu\left(C_{n}^{\prime \prime \prime}+\gamma_{n}^{\prime \prime \prime}\right), \\
& A_{n}^{* *}(r, \mu, \phi)=\mu\left(A_{n}^{\prime}+\alpha_{n}^{\prime}\right) \cos \phi+\mu\left(A_{n}^{\prime \prime}+\alpha_{n}^{\prime \prime}\right) \sin \phi-\left(1-\mu^{2}\right)^{1 / 2}\left(A_{n}^{\prime \prime \prime}+\alpha_{n}^{\prime \prime \prime}\right),
\end{aligned}
$$




$$
\begin{aligned}
& B_{n}^{* *}(r, \mu, \phi)=\mu\left(B_{n}^{\prime}+\beta_{n}^{\prime}\right) \cos \phi+\mu\left(B_{n}^{\prime \prime}+\beta_{n}^{\prime \prime}\right) \sin \phi-\left(1-\mu^{2}\right)^{1 / 2}\left(B_{n}^{\prime \prime \prime}+\beta_{n}^{\prime \prime \prime}\right), \\
& C_{n}^{* *}(r, \mu, \phi)=\mu\left(C_{n}^{\prime}+\gamma_{n}^{\prime}\right) \cos \phi+\mu\left(C_{n}^{\prime \prime}+\gamma_{n}^{\prime \prime}\right) \sin \phi-\left(1-\mu^{2}\right)^{1 / 2}\left(C_{n}^{\prime \prime \prime}+\gamma_{n}^{\prime \prime \prime}\right), \\
& A_{n}^{* * *}(r, \mu, \phi)=-\left(A_{n}^{\prime}+\alpha_{n}^{\prime}\right) \sin \phi+\left(A_{n}^{\prime \prime}+\alpha_{n}^{\prime \prime}\right) \cos \phi, \\
& B_{n}^{* * *}(r, \mu, \phi)=-\left(B_{n}^{\prime}+\beta_{n}^{\prime}\right) \sin \phi+\left(B_{n}^{\prime \prime}+\beta_{n}^{\prime \prime}\right) \cos \phi, \\
& C_{n}^{* * *}(r, \mu, \phi)=-\left(C_{n}^{\prime}+\gamma_{n}^{\prime}\right) \sin \phi+\left(C_{n}^{\prime \prime}+\gamma_{n}^{\prime \prime}\right) \cos \phi .
\end{aligned}
$$

Here, the primed $A_{n}, B_{n}, C_{n}, \alpha_{n}, \beta_{n}$, and $\gamma_{n}$ are functions of position in (18) and (22).

\section{References}

Acrivos, A., Jeffrey, D.J., Saville, D.A., 1990. Particle migration in suspensions by thermocapillary or electrophoretic motion. J. Fluid Mech. 212, 95-110.

Anderson, J.L., 1985. Droplet interactions in thermocapillary motion. Int. J. Multiphase Flow 11, 813-824.

Ascoli, E.P., Leal, L.G., 1990. Thermocapillary motion of a deformable drop toward a planar wall. J. Colloid Interf. Sci. $138,220-230$.

Barton, K.D., Subramanian, R.S., 1990. Thermocapillary migration of a liquid drop normal to a plane surface. J. Colloid Interf. Sci. 137, 170-182.

Barton, K.D., Subramanian, R.S., 1991. Migration of liquid drops in a vertical temperature gradient-interaction effects near a horizontal surface. J. Colloid Interf. Sci. 141, 146-156.

Chen, J., Dagan, Z., Maldarelli, C., 1991. The axisymmetric thermocapillary motion of a fluid particle in a tube. J. Fluid Mech. 233, 405-437.

Chen, S.H., 1999. Thermocapillary deposition of a fluid droplet normal to a planar surface. Langmuir 15, 2674-2683.

Chen, S.H., Keh, H.J., 1990. Thermocapillary motion of a fluid droplet normal to a plane surface. J. Colloid Interf. Sci. $137,550-562$.

Ganatos, P., Weinbaum, S., Pfeffer, R., 1980. A strong interaction theory for the creeping motion of a sphere between plane parallel boundaries. Part 2. Parallel motion. J. Colloid Interf. Sci. 99, 755-783.

Happel, J., Brenner, H., 1983. Low Reynolds Number Hydrodynamics. Nijhoff, The Hague, The Netherlands.

Hetsroni, G., Haber, S., 1970. The flow in and around a droplet or bubble submerged in an unbound arbitrary velocity field. Rheol. Acta. 9, 488-496.

Kasumi, H., Solomentsev, Y.E., Guelcher, S.A., Anderson, J.L., Sides, P.J., 2000. Thermocapillary flow and aggregation of bubbles on a solid wall. J. Colloid Interf. Sci. 232, 111-120.

Keh, H.J., Chen, L.S., 1993. Droplet interactions in thermocapillary migration. Chem. Engng. Sci. 48, 3565-3582.

Keh, H.J., Chen, P.Y., 2001. Slow motion of a droplet between two parallel plane walls. Chem. Engng. Sci. 56, 68636871.

Loewenberg, M., Davis, R.H., 1993a. Near-contact thermocapillary motion of two non- conducting drops. J. Fluid Mech. 256, 107-131.

Loewenberg, M., Davis, R.H., 1993b. Near-contact, thermocapillary migration of a nonconducting, viscous drop normal to a planar interface. J. Colloid Interf. Sci. 160, 265-274.

Meyyappan, M., Subramanian, R.S., 1987. Thermocapillary migration of a gas bubble in an arbitrary direction with respect to a plane surface. J. Colloid Interf. Sci. 115, 206-219.

Meyyappan, M., Wilcox, W.R., Subramanian, R.S., 1981. Thermocapillary migration of a bubble normal to a plane surface. J. Colloid Interf. Sci. 83, 199-208.

Meyyappan, M., Wilcox, W.R., Subramanian, R.S., 1983. The slow axisymmetric motion of two bubbles in a thermal gradient. J. Colloid Interf. Sci. 94, 243-257.

Morton, D.S., Subramanian, R.S., Balasubramaniam, R., 1990. The migration of a compound drop due to thermocapillarity. Phys. Fluids A 2, 2119-2133.

O'Brien, V., 1968. Form factors for deformed spheroids in Stokes flow. AIChE J. 14, 870-875. 
Sadhal, S.S., 1983. A note on the thermocapillary migration of a bubble normal to a plane surface. J. Colloid Interf. Sci. 95, 283-285.

Satrape, J.V., 1992. Interactions and collisions of bubbles in thermocapillary motion. Phys. Fluids A 4, 1883-1900.

Young, N.O., Goldstein, J.S., Block, M.J., 1959. The motion of bubbles in a vertical temperature gradient. J. Fluid Mech. 6, 350-364. 